\title{
Theory of Orbital State and Spin Interactions in Ferromagnetic Titanates
}

\author{
Giniyat Khaliullin \\ Max-Planck-Institut für Festkörperforschung, Heisenbergstrasse 1, D-70569 Stuttgart, Germany \\ Satoshi Okamoto* \\ The Institute of Physical and Chemical Research (RIKEN), Saitama 351-0198, Japan
}

(November 10, 2018)

\begin{abstract}
A spin-orbital superexchange Hamiltonian in a Mott insulator with $t_{2 g}$ orbital degeneracy is investigated. More specifically, we focus on a spin ferromagnetic state of the model and study a collective behavior of orbital angular momentum. Orbital order in the model occurs in a nontrivial way it is stabilized exclusively by quantum effects through the order-from-disorder mechanism. Several energetically equivalent orbital orderings are identified. Some of them are specified by a quadrupole ordering and have no unquenched angular momentum at low energy. Other states correspond to a noncollinear ordering of the orbital angular momentum and show the magnetic Bragg peaks at specific positions. Order parameters are unusually small because of strong quantum fluctuations. Orbital contribution to the resonant x-ray scattering is discussed. The dynamical magnetic structure factor in different ordered states is calculated. Predictions made should help to observe elementary excitations of orbitals and also to identify the type of the orbital order in ferromagnetic titanates. Including further a relativistic spin-orbital coupling, we derive an effective low-energy spin Hamiltonian and calculate a spin-wave spectrum, which is in good agreement with recent experimental observations in $\mathrm{YTiO}_{3}$.
\end{abstract}

PACS numbers: 75.10.-b, 75.30.Ds, 75.30.Et, 78.70.Nx

\section{INTRODUCTION}

Many transition-metal oxides fall into the category of Mott insulators, ${ }^{1}$ in which the large degeneracy of atomic states remain unquenched down to low energies. Of particular importance here is the role being played by orbital degeneracy inherent to perovskite lattices. An additional degeneracy of low-energy states and the extreme sensitivity of the chemical bonds to the spatial orientation of orbitals lead to frustrating interactions and a variety of competing phases that are tunable by moderate external fields. ${ }^{2}$

As "orbital physics" has started to become an essential ingredient of the physics of transition-metal oxides, more efforts are necessary to develop quantum many-body theory of coupled spin-orbital systems in order to understand specific features of the orderings and fluctuations in these models. Earlier work has emphasized a "classical part" of the problem, focusing mainly on the strong interplay between classical spin and orbital configurations. It is implicitly assumed that at low temperature orbitals are frozen in a certain static pattern that optimizes both superexchange (SE) and orbital-lattice (JT) couplings. Such a classical approach has been used with a great success as a theoretical guide in studies of magnetism of transition-metal oxides.

Recent experimental developments indicate, however, the limitations of this standard picture. It has been argued that quantum fluctuations of orbitals might sometimes be of crucial importance, hence quantum version of the orbital physics is needed. New concepts, such as three-dimensional orbital liquid in $\mathrm{LaTiO}_{3}$ (Refs. 3 and onlineciteKHA00) and one-dimensional orbital chains showing Heisenberg-like orbital dynamics in cubic vanadates (Ref. 5), have been proposed. It is not accidental that these ideas emerge from a study of titanates and vanadates having $t_{2 g}^{1}$ and $t_{2 g}^{2}$ electronic configurations, respectively. This is because of (i) large, threefold degeneracy, and (ii) a special rotational symmetry of $t_{2 g}$ orbitals. Another crucial point is that (iii) the JT coupling is relatively weak for $t_{2 g}$ systems. Indeed, JT-like elongation of octahedra on titanates and vanadates is much smaller compared with typical JT distortions in manganites with $e_{g}$ orbitals. One may therefore think that $t_{2 g}$ orbital states are much less affected by electron-lattice coupling, and an intrinsic dynamics of coupled spin-orbital system governed by electronic superexchange interaction becomes the decisive factor in a first place. Effects of lattice distortions (which are always present) can then be accounted for in a next step. This point has actually been emphasized long ago by Kugel and Khomskii ${ }^{6}$ indicating also very peculiar specific features of $t_{2 g}$ spinorbital models. ${ }^{7}$

The aim of this paper is twofold. First, we study the orbital state and orbital quantum dynamics in the ferromagnetic state of superexchange model with $t_{2 g}$ orbital degeneracy in a cubic lattice. Second, we discuss the results in context of the magnetic properties of $\mathrm{YTiO}_{3}$, a rare example of a ferromagnetic Mott insulator. ${ }^{8,9}$ Recent spin-wave data shows that the ferromagnetic (F) state of this material is highly isotropic having the same exchange couplings in all cubic directions. ${ }^{10}$ This is in sharp contrast with expectations from the conventional orbital ordering picture resulting commonly in a strong spatial anisotropy of the spin exchange bonds. ${ }^{11,12}$ This observation already indicates a rather unusual orbital state in 
$\mathrm{YTiO}_{3}$. We would like also to understand a mechanism which stabilizes such a isotropic F-state in $\mathrm{YTiO}_{3}$, having in mind that its sister compound, $\mathrm{LaTiO}_{3}$ shows a completely different, antiferromagnetic ( $\mathrm{AF}$ ) state. $\mathrm{Cu}-$ riously enough, spin-exchange couplings in $\mathrm{LaTiO}_{3}$ are also of cubic symmetry and spin gap is also small, ${ }^{3}$ and these observations were understood in terms of fluctuating orbitals. ${ }^{4,13}$

We argue that $\mathrm{AF}$ and $\mathrm{F}$ states in $t_{2 g} \mathrm{SE}$ model are actually very close in energy and strongly compete. This is because in both states there are large-scale orbital fluctuations gaining almost the same amount of the superexchange energy. Yet the AF state is slightly lower because of an additional, composite spin-orbital fluctuation. However, an external parameter, namely, a larger distortion of Ti-O-Ti bonds due to a small size of $\mathrm{Y}$-ion in case $\mathrm{YTiO}_{3}$ induces an additional ferromagnetic coupling in all three directions, and stabilizes the spin F state. This distortion induces also a gap for orbital excitations. The orbital order pattern is very specific, and it supports exactly the same ferrocouplings in all three directions. We derive an effective spin Hamiltonian which includes effects of the relativistic spin-orbital coupling as well, and show that this Hamiltonian leads to spin-wave dispersion and spin gap consistent with experimental observations. Some of these results were presented in Ref. 14 .

The following part of this paper is structured as follows: Section II presents superexchange Hamiltonian in $t_{2 g}^{1}$ Mott insulator; Sec. III presents orbital ordering and fluctuations in the F state; Sec. IV presents stabilization of the F state by Ti-O-Ti bond distortion; Sec. V presents orbital gap induced by $\mathrm{Ti}-\mathrm{O}-\mathrm{Ti}$ bond distortion; Effective spin Hamiltonian and magnons are given in Sec. (VI); Sec. VII lists predictions for resonant $\mathrm{x}-$ ray scattering; Sec. VIII gives orbital angular momentum contribution to the neutron scattering cross-section; (IX) Summary and discussion. The appendixes A-C contain some lengthy equations; Appendix D shows magnon softening by orbital fluctuations; Appendix E shows spin interactions in a previously reported orbital state for $\mathrm{YTiO}_{3}$.

\section{HAMILTONIAN}

\section{A. Superexchange interaction in $t_{2 g}$ orbital system}

We start with a discussion of the model Hamiltonian. In Mott insulators, the competition between kinetic and potential energies is resolved in favor of strong correlations that lead to a localized electron picture. Charge localization is however not perfect: electrons still make virtual excursions to neighboring sites in order to retain their kinetic energy at least partially. In terminology of Mott-Hubbard insulators, ${ }^{1}$ the zero-point charge motion is described as a high-energy virtual transition across the Mott gap. Kinetic energy associated with these transi- tions leads to superexchange interactions, which in orbitally degenerate systems strongly depends on the orbital structure. In general, it can be written as

$$
H_{S E}^{i j}=\left(\vec{S}_{i} \cdot \vec{S}_{j}+\frac{1}{4}\right) \hat{J}_{i j}^{(\gamma)}+\frac{1}{2} \hat{K}_{i j}^{(\gamma)}
$$

where the orbital operators $\hat{J}_{i j}^{(\gamma)}$ and $\hat{K}_{i j}^{(\gamma)}$ depend on bond directions $\gamma(=a, b, c)$. In a $t_{2 g}$ system like the titanates they are given by the following expressions: ${ }^{13}$

$$
\begin{aligned}
\hat{J}_{i j}^{(\gamma)}=J_{S E}[ & \frac{1}{2}\left(r_{1}+r_{2}\right) A_{i j}^{(\gamma)}-\frac{1}{3}\left(r_{2}-r_{3}\right) B_{i j}^{(\gamma)} \\
& \left.-\frac{1}{4}\left(r_{1}-r_{2}\right)\left(n_{i}+n_{j}\right)^{(\gamma)}\right], \\
\hat{K}_{i j}^{(\gamma)}=J_{S E}[ & \frac{1}{2}\left(r_{1}-r_{2}\right) A_{i j}^{(\gamma)}+\frac{1}{3}\left(r_{2}-r_{3}\right) B_{i j}^{(\gamma)} \\
& \left.-\frac{1}{4}\left(r_{1}+r_{2}\right)\left(n_{i}+n_{j}\right)^{(\gamma)}\right],
\end{aligned}
$$

where $J_{S E}=4 t^{2} / U$. The coefficients $r_{1}=1 /(1-3 \eta)$, $r_{2}=1 /(1-\eta)$, and $r_{3}=1 /(1+2 \eta)$ originate from the Hund's splitting of the excited $t_{2 g}^{2}$ multiplet via $\eta=J_{H} / U$. Reference 15 gives $J_{H} \sim 0.64 \mathrm{eV}$ and the multiplet averaged Coulomb interaction $\left(=U-\frac{20}{9} J_{H}\right) \sim$ $4 \mathrm{eV}$, from which representative values $U \sim 5.4 \mathrm{eV}$ and $\eta \sim 0.12$ follow.

The operators $A_{i j}^{(\gamma)}, B_{i j}^{(\gamma)}$, and $n_{i}^{(\gamma)}$ can conveniently be represented in terms of constrained particles (orbitons) $a_{i}, b_{i}, c_{i}$ with $n_{i a}+n_{i b}+n_{i c}=1$ corresponding to $t_{2 g}$ levels of $y z, x z, x y$ symmetry, respectively. (This notation is motivated by the fact that each $t_{2 g}$ orbital is orthogonal to one of the cubic axes $a, b, c$.) Namely,

$$
\begin{aligned}
& A_{i j}^{(c)}=n_{i a} n_{j a}+n_{i b} n_{j b}+a_{i}^{\dagger} b_{i} b_{j}^{\dagger} a_{j}+b_{i}^{\dagger} a_{i} a_{j}^{\dagger} b_{j}, \\
& B_{i j}^{(c)}=n_{i a} n_{j a}+n_{i b} n_{j b}+a_{i}^{\dagger} b_{i} a_{j}^{\dagger} b_{j}+b_{i}^{\dagger} a_{i} b_{j}^{\dagger} a_{j}, \\
& n_{i}^{(c)}=n_{i a}+n_{i b}
\end{aligned}
$$

for the pair along the $c$ axis. Similar expressions are obtained for the exchange bonds along the axes $a$ and $b$, by replacing orbitons $(a, b)$ in Eqs. (4)-(6) by $(b, c)$ and $(c, a)$ pairs, respectively. ${ }^{16}$ Another useful representation of orbital exchange operators is via the angular momentum operators of $t_{2 g}$ level, ${ }^{7}$ using the following relations:

$$
l_{x}=i\left(c^{\dagger} b-b^{\dagger} c\right), l_{y}=i\left(a^{\dagger} c-c^{\dagger} a\right), l_{z}=i\left(b^{\dagger} a-a^{\dagger} b\right) .
$$

In terms of these angular momentum operators, $A_{i j}^{(\gamma)}$, $B_{i j}^{(\gamma)}$, and $n_{i}^{(\gamma)}$ are represented as

$$
\begin{aligned}
& A_{i j}^{(c)}=\left[\left(1-l_{x}^{2}\right)_{i}\left(1-l_{x}^{2}\right)_{j}+\left(l_{x} l_{y}\right)_{i}\left(l_{y} l_{x}\right)_{j}\right]+[x \leftrightarrow y], \\
& B_{i j}^{(c)}=\left[\left(1-l_{x}^{2}\right)_{i}\left(1-l_{x}^{2}\right)_{j}+\left(l_{x} l_{y}\right)_{i}\left(l_{x} l_{y}\right)_{j}\right]+[x \leftrightarrow y], \\
& n_{i}^{(c)}=l_{i z}^{2} .
\end{aligned}
$$

Expressions of these operators for $a$ and $b$ bonds are given by replacing two component of the angular momentum 
$\left(l_{x}, l_{y}\right)$ in Eqs. (8)-(10) with $\left(l_{y}, l_{z}\right)$ and $\left(l_{z}, l_{x}\right)$, respectively. Angular and quadrupole momentum representation of the $t_{2 g}$ superexchange has recently been used also in Ref. 17. In addition to Eqs. (4)-(5) and (8)-(9), it is also useful to represent $A_{i j}^{(\gamma)}$ and $B_{i j}^{(\gamma)}$ in terms of auxiliary orbital pseudospins:

$$
\begin{aligned}
& A_{i j}^{(\gamma)}=2\left(\vec{\tau}_{i} \cdot \vec{\tau}_{j}+\frac{n_{i} n_{j}}{4}\right)^{(\gamma)}, \\
& B_{i j}^{(\gamma)}=2\left(\vec{\tau}_{i} \otimes \vec{\tau}_{j}+\frac{n_{i} n_{j}}{4}\right)^{(\gamma)} .
\end{aligned}
$$

Here $\vec{\tau}_{i}^{(\gamma)}$ is a pseudospin one-half operating on the subspace of orbital doublet $(\alpha, \beta)^{(\gamma)}$ active on a given $\gamma$ bond. Namely, pseudospin $\vec{\tau}_{i}^{(c)}$ operates on the subspace spanned by $(a, b)$ pair of orbitons, while $\vec{\tau}_{i}^{(a)}$ and $\vec{\tau}_{i}^{(b)}$ act on $(b, c)$ and $(c, a)$ doublets, respectively. A symbol $\otimes$ denotes a product $\vec{\tau}_{i} \otimes \vec{\tau}_{j}=\tau_{i}^{z} \tau_{j}^{z}+\left(\tau_{i}^{+} \tau_{j}^{+}+\tau_{i}^{-} \tau_{j}^{-}\right) / 2$.

\section{B. Ferromagnetic state}

The ferromagnetic state of a Mott insulator is usually thought due to a particular orbital ordering that optimizes the intraatomic Hund's exchange of electrons in doubly occupied virtual states. This is not the whole story, however. Neglect for a moment the Hund's coupling terms in Eqs.(2) and (3) (consider $\eta \rightarrow 0$ limit). The Hamiltonian obtains then the following structure:

$$
H_{0}=J_{S E} \sum_{\langle i j\rangle} 2\left(\vec{S}_{i} \cdot \vec{S}_{j}+\frac{1}{4}\right)\left(\vec{\tau}_{i} \cdot \vec{\tau}_{j}+\frac{1}{4} n_{i} n_{j}\right)^{(\gamma)} .
$$

(The unessential energy shift, $-J_{S E}$, is not shown here). Regarding a single bond, one notices that spin coupling may be of either sign, depending on the intersite orbital correlations. Singlet correlations of orbital pseudospins tend to align spins ferromagnetically, hence cooperating with Hund's rule effects. In systems with large, classical spins (e.g., vanadates), such a quantum orbital singlet controls the ground state. ${ }^{5,18}$ In quantum spin one-half case of titanates, however, (spin triplet) $\times$ (orbital singlet) and (spin singlet) $\times$ (orbital triplet) configurations are degenerate and compete. In a lattice, quantum resonances between these configurations are possible. ${ }^{4}$ In general, $t_{2 g}$ superexchange Hamiltonian Eq. (1) represents a highly frustrated many-body problem. We will return to the interplay between antiferromagnetic and ferromagnetic states later on, while focusing now on the ferromagnetic state realized in $\mathrm{YTiO}_{3}$.

In the spin saturated state, Eqs. (1)-(3) are simplified to:

$$
H_{o r b}=-r_{1} J_{S E}+\frac{1}{2} r_{1} J_{S E} \sum_{\langle i j\rangle} A_{i j}^{(\gamma)}
$$

where $A_{i j}^{(\gamma)}$ is given by either of Eqs.(4),(8), and (11). We consider the orbital order and dynamics in this Hamiltonian. The effects of the dynamical coupling between spin excitations and orbitals that is present in Eq. (1) will also be discussed in the context of magnon spectra.

\section{ORBITAL ORDERING AND EXCITATIONS}

\section{A. Discussion of possible orderings}

Even though spin as well as composite spin/orbital dynamics is "switched off" in Hamiltonian Eq. (14), it still contains nontrivial physics.

It is useful to look at the structure of $H_{\text {orb }}$ from different points of view. (i) On a given bond, the operator $A_{i j}^{(\gamma)}$ acts within a particular doublet of equivalent orbitals. Spin-like physics, that is the formation of orbital singlets is therefore possible. (ii) On the other hand, interactions on different bonds are competing: they involve different doublets, thus frustrating each other. This brings about a Potts-model-like frustration, from which the high degeneracy of classical orbital configurations follows. (iii) Finally, we observe in $A_{i j}^{(\gamma)}$ a pseudospin $l=1$ interaction of pure biquadratic form [see Eq. (8)]. Would $\vec{l}$ be a classical vector, it could change its sign at any site independently. Such a local (so-called $Z_{2}$ ) symmetry and the associated degeneracy of the classical states tell us that angular momentum ordering, if any, must be of pure quantum origin.

The above points (i)-(iii) govern the underlying physics of the orbital Hamiltonian. We need to find such classical states that provide best zero point energy when we switch on the quantum fluctuations. In other words, certain classical orbital patterns will be selected and stabilized by quantum effects via the order-from-disorder mechanism. ${ }^{19}$ Normally, these orderings are expected to be along symmetric orientations of the crystal depending on symmetry of the underlying interactions.

By inspection of the global structure of $A_{i j}^{(\gamma)}$ [Eq. (8)], one observes that the non-cross terms, such as $(1-$ $\left.l_{x}^{2}\right)_{i}\left(1-l_{x}^{2}\right)_{j}$, are definitely positive. However, the cross terms, $\left(l_{x} l_{y}\right)_{i}\left(l_{y} l_{x}\right)_{j}$ and $\left(l_{y} l_{x}\right)_{i}\left(l_{x} l_{y}\right)_{j}$ [which change the "color" of orbitals, see Eq. (4)], can be made negative on all the bonds simultaneously, if (i) on every bond, two particular components of $\vec{l}_{i}$ and $\vec{l}_{j}$ are antiparallel, and (ii) remaining third components are parallel. For $c$ bonds the rule reads as: $l_{i z} l_{j z}$ and $l_{i x} l_{j x}$ are both negative, while $l_{y}$ components are parallel. (In terms of orbitons: $c_{i}$ and $c_{j}$ are in antiphase, $a_{i}$ and $a_{j}$ as well; but $b_{i}$ and $b_{j}$ have the same phase.) We find only two topologically different arrangements [called (a) and (b)], which can accommodate this curious mixture of " $2 / 3$ antiferro" plus " $1 / 3$ ferro" correlations (see Figs. 1 and 2). In the state (a), sublattice unit vectors are along the cubic diagonals [111], 


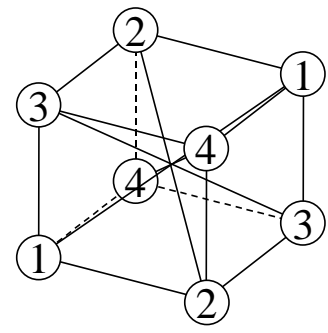

(a)

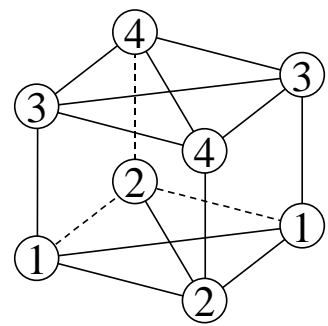

(b)
FIG. 1. Two kinds of four-sublattice structure for orbital orderings in spin ferromagnetic $t_{2 a}$ superexchange model.

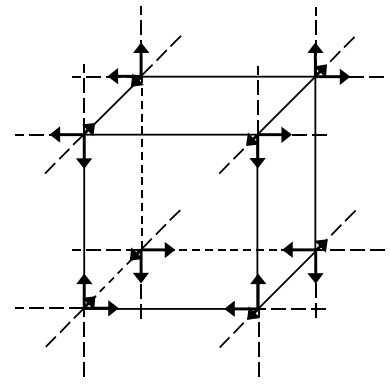

(a)

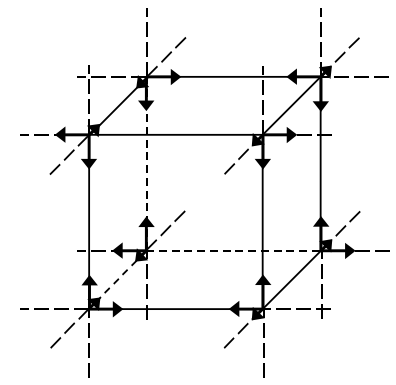

(b)
FIG. 2. Arrangement of the local quantization axes in states (a) and (b). Arrows indicate the quantization axes at each site, and represent also a snapshot of local correlations of angular momentum: on every bond, two out of three components of $\vec{l}$ are correlated antiparallel.

while in state (b), sublattice unit vectors are [110] and [002].

For technical reasons, it is useful to introduce new quantization axes. This is done in two steps. First, we introduce local, sublattice specified quantization axes (see Fig. 2):

$$
\begin{aligned}
& 1:(x, y, z) \rightarrow(x, y, z), \\
& 2:(x, y, z) \rightarrow(-x,-y, z), \\
& 3:(x, y, z) \rightarrow(-x, y,-z), \\
& 4:(x, y, z) \rightarrow(x,-y,-z) .
\end{aligned}
$$

After corresponding sign transformations of $l_{i \alpha}$ and orbitons, one obtains

$$
\begin{aligned}
A_{i j}^{(c)} & =n_{i a} n_{j a}+n_{i b} n_{j b}-a_{i}^{\dagger} b_{i} b_{j}^{\dagger} a_{j}-b_{i}^{\dagger} a_{i} a_{j}^{\dagger} b_{j} \\
& =\left[\left(1-l_{x}^{2}\right)_{i}\left(1-l_{x}^{2}\right)_{j}-\left(l_{x} l_{y}\right)_{i}\left(l_{y} l_{x}\right)_{j}\right]+[x \leftrightarrow y] .
\end{aligned}
$$

¿From now on, a sublattice structure will not enter in the excitation spectrum. From the above observations it is also clear that all the components of $\vec{l}$ are equally needed to optimize all the three directions. We anticipate therefore that the cubic diagonals are "easy" (or "hard") axes for $\vec{l}$ fluctuations/orderings (recall that the Hamiltonian has no rotational symmetry for $\vec{l}$ vector). Therefore, it is convenient to further rotate the quantization axis so that new $z$-axis (denoted as $\tilde{z}$ ) corresponds to [111] direction. This is done as follows:

$$
\vec{l}_{i}=\hat{R} \overrightarrow{\tilde{l}}_{i}
$$

where $\overrightarrow{\tilde{l}}_{i}=\left(\tilde{l}_{i x}, \tilde{l}_{i y}, \tilde{l}_{i z}\right)$ and $\hat{R}$ is given by

$$
\hat{R}=\frac{1}{\sqrt{3}}\left(\begin{array}{ccc}
c+s & c-s & 1 \\
c-s & c+s & 1 \\
-1 & -1 & 1
\end{array}\right),
$$

with $c=1 / 2$ and $s=\sqrt{3} / 2$. Here, new $\tilde{x}$ - and $\tilde{y}$-axes are taken to be symmetric with respect to the [110] direction. Annihilation operators for constrained particles obey the same transformation,

$$
\left(\begin{array}{c}
a \\
b \\
c
\end{array}\right)=\hat{R}\left(\begin{array}{c}
\tilde{a} \\
\tilde{b} \\
\tilde{c}
\end{array}\right) .
$$

Explicit expressions for the wave functions $\psi_{\tilde{\alpha}}$ are obtained by reversing Eq. (20) as follows:

$$
\begin{aligned}
\psi_{\tilde{a}} & =\frac{1}{\sqrt{3}}\left[(c+s) d_{y z}+(c-s) d_{x z}-d_{x y}\right], \\
\psi_{\tilde{b}} & =\frac{1}{\sqrt{3}}\left[(c-s) d_{y z}+(c+s) d_{x z}-d_{x y}\right], \\
\psi_{\tilde{c}} & =\frac{1}{\sqrt{3}}\left(d_{y z}+d_{x z}+d_{x y}\right) .
\end{aligned}
$$

In Fig. 3, we show schematic pictures of these orbitals. By construction, $\psi_{\tilde{c}}$ is symmetric with respect to the rotation around [111] direction, having simply $3 \tilde{z}^{2}-r^{2}$ symmetry, while $\psi_{\tilde{a}}$ and $\psi_{\tilde{b}}$ are symmetric with respect to [110] axis. At the end, the orbital Hamiltonian in a rotated basis obtains the following form (symbol "tilde" denoting rotated axes is implied for angular and quadrupole operators below, and constant energy shift is dropped out):

$$
H_{\text {orb }}=\frac{1}{2} r_{1} J_{S E} \sum_{\langle i j\rangle} A_{i j}^{(\gamma)}
$$

with

$$
\begin{aligned}
3 A_{i j}^{(\gamma)}= & \frac{2}{3}\left(1-Q_{i z} Q_{j z}\right)-\frac{1}{2} l_{i z} l_{j z} \\
& +\frac{1}{2}\left(Q_{x} T_{-1}+T_{-1} Q_{x}-T_{0} T_{1}-T_{1} T_{0}\right)_{i j} \\
& +\frac{1}{2}\left(Q_{x} Q_{x}-T_{0} T_{0}+T_{-1} T_{-1}\right)_{i j}^{(\gamma)} \\
& +\frac{2}{3} Q_{i z}\left(T_{0}+c T_{1}\right)_{j}^{(\gamma)}+\frac{2}{3}\left(T_{0}+c T_{1}\right)_{i}^{(\gamma)} Q_{j z} \\
& +\frac{1}{2} l_{i z}\left(l_{x}+l_{y}\right)_{j}^{(\gamma)}+\frac{1}{2}\left(l_{x}+l_{y}\right)_{i}^{(\gamma)} l_{j z} \\
& -\frac{1}{2}\left(l_{x}+l_{y}\right)_{i}^{(\gamma)}\left(l_{x}+l_{y}\right)_{j}^{(\gamma)},
\end{aligned}
$$

where $Q_{z}$ and $Q_{x}$ represent the quadrupole moment operators with $e_{g}$ symmetry, $3 z^{2}-r^{2}$ and $x^{2}-y^{2}$, respectively. $T_{0}=T_{z}$ and $T_{ \pm 1}=T_{y} \pm T_{x}$, where $T_{z}, T_{y}$, and 


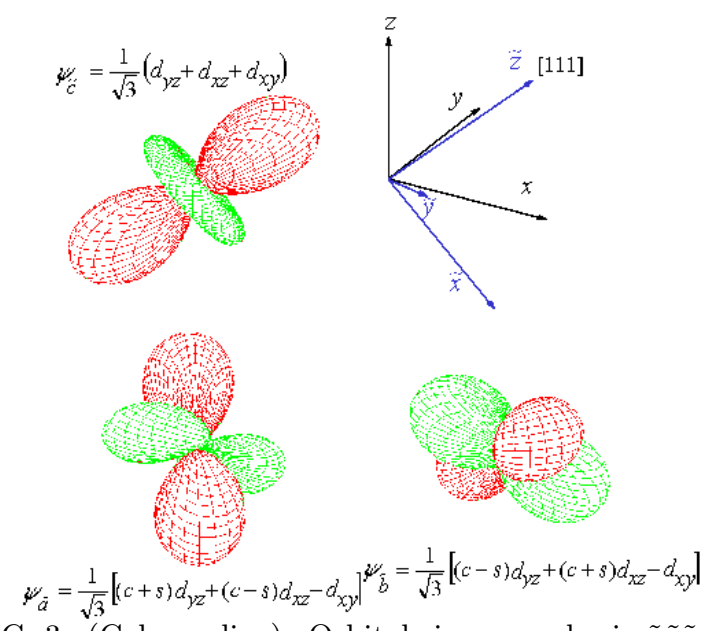

FIG. 3. (Color online). Orbitals in a new basis $\tilde{x} \tilde{y} \tilde{z}$ specified by transformation Eq. (19).

$T_{x}$ represent the quadrupole moment operators with $t_{2 g}$ symmetry of $x y, x z$, and $y z$, respectively. These operators are expressed in terms of angular momentum and orbiton operators as follows:

$$
\begin{aligned}
Q_{z} & =\frac{1}{2}\left(\vec{l}^{2}-3 l_{z}^{2}\right)=n_{\tilde{c}}-\frac{1}{2}\left(n_{\tilde{a}}+n_{\tilde{b}}\right), \\
Q_{x} & =l_{x}^{2}-l_{y}^{2}=n_{\tilde{b}}-n_{\tilde{a}}, \\
T_{z} & =l_{x} l_{y}+l_{y} l_{x}=-\left(\tilde{a}^{\dagger} \tilde{b}+\tilde{b}^{\dagger} \tilde{a}\right), \\
T_{x} & =l_{y} l_{z}+l_{z} l_{y}=-\left(\tilde{b}^{\dagger} \tilde{c}+\tilde{c}^{\dagger} \tilde{b}\right), \\
T_{y} & =l_{x} l_{z}+l_{z} l_{x}=-\left(\tilde{c}^{\dagger} \tilde{a}+\tilde{a}^{\dagger} \tilde{c}\right) .
\end{aligned}
$$

$\gamma$-dependence of quadrupole moment operators is obtained by changing $l_{x, y}$ in Eq. (24) to

$$
\begin{aligned}
& l_{x}^{(\gamma)}=\left\{\begin{aligned}
-c l_{x} \pm s l_{y}, & \text { for } \gamma=a(b) \\
l_{x}, & \text { for } \gamma=c
\end{aligned}\right. \\
& l_{y}^{(\gamma)}=\left\{\begin{aligned}
-c l_{y} \mp s l_{x}, & \text { for } \gamma=a(b) \\
l_{y} . & \text { for } \gamma=c
\end{aligned}\right.
\end{aligned}
$$

Explicit expressions for $Q_{\alpha}^{(\gamma)}$ and $T_{\alpha}^{(\gamma)}$ are given in Appendix A. It should be noted that, among eight operators, namely, five quadrupole moment and three angular momentum operators, only four operators are independent of each other because of the local constraint among orbiton operators.

Although it looks a bit complicated, the rotated Hamiltonian obtains a well-structured form. The first and second terms of Eq. (23) represent "Ising"-like interaction for quadrupole moments and angular momenta. This part of the Hamiltonian stabilizes the ordering (condensation) of an appropriate orbiton. On the other hand, the other terms represent fluctuations of $Q_{x}, T_{x}, T_{y}, T_{z}$ and transverse components of angular momenta $l_{x}$ and $l_{y}$. These terms generate dispersion of the orbital excitations.

Ordered states, promoted by the "Ising" part of interactions, can be characterized by the quadrupole moment
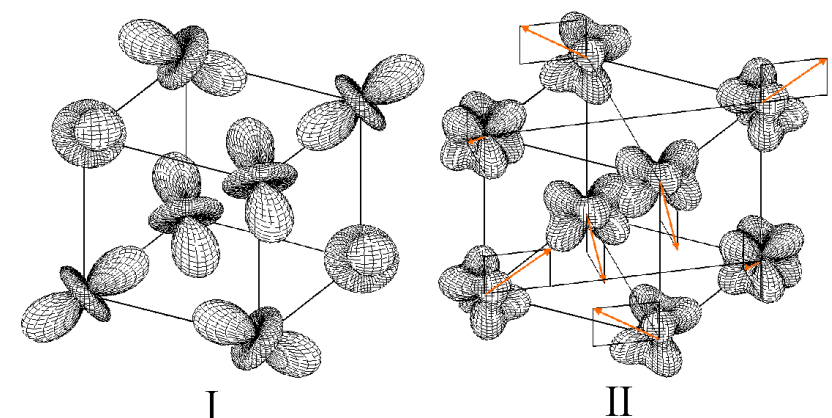

FIG. 4. (Color online). Schematic pictures of the orbital orderings. Left: Real orbital ordering I(a). Right: Complex orbital ordering II(a). Here, the absolute values of the wave functions are presented. Arrows represent the directions of angular magnetic momenta in the orbital magnetic state II(a).

$Q=\left\langle Q_{z}\right\rangle$ (Q-order may couple to a lattice distortion of $D_{3 d}$ symmetry), and the angular magnetic moment $m_{l}=\left\langle\tilde{l}_{z}\right\rangle$. We notice that the magnetic, $l_{i z} l_{j z}$ term in the first line of Eq. (23) is generated by quantum commutation rules when we rotate $H_{\text {orb }}$; this makes explicit that the $Z_{2}$ symmetry is only a classical one and emphasizes the quantum origin of orbital magnetism.

As it follows from the definition of $Q_{z}$, quadrupole ordering with finite $Q$ but zero $l_{z}$ corresponds to a condensation of the $\tilde{c}$ orbiton. We call this solution state I. Classically, $Q=1$ in this state. On the other hand, condensation of the complex orbital $(\tilde{a}-i \tilde{b}) / \sqrt{2}$ generates a finite magnetic moment $\left(m_{l}=1\right.$ classically), the state called II. The orbital patterns in a classical states I and II are shown in Fig. 4. We now focus on fluctuations of orbitals, and show that the excitation spectra are in fact identical in these states. Moreover, we will obtain that the states I and II can smoothly be connected by a continuous phase rotation of the condensate wave function. Noticing that an arbitrary cubic diagonal could be taken as $\tilde{z}$ and having in mind also two structures in Fig. 1, one obtains a multitude of degenerate states. This makes, in fact, all the orderings very fragile.

\section{B. Orbital quadrupole order}

This state is driven by a condensation of $\tilde{c}$ orbital, that is, ordering of the orbital $\psi_{\tilde{c}}$ in Eq.(21). To obtain a linear orbital wave Hamiltonian, we resolve a constraint as $\tilde{c}=\tilde{c}^{\dagger}=\sqrt{1-n_{\tilde{a}}-n_{\tilde{b}}}$, and expand Eq. (23) up to second order in $\tilde{a}$ and $\tilde{b}$. The result is (in units of $r_{1} J_{S E}$ ):

$$
\begin{aligned}
H_{O W} & =\sum_{i}\left(n_{i \tilde{a}}+n_{i \tilde{b}}\right) \\
& +\frac{1}{2 z} \sum_{\langle i j\rangle}\left[T_{i-1}^{(\gamma)} T_{j-1}^{(\gamma)}-\left(l_{x}+l_{y}\right)_{i}^{(\gamma)}\left(l_{x}+l_{y}\right)_{j}^{(\gamma)}\right],
\end{aligned}
$$

where $z=6$, and 

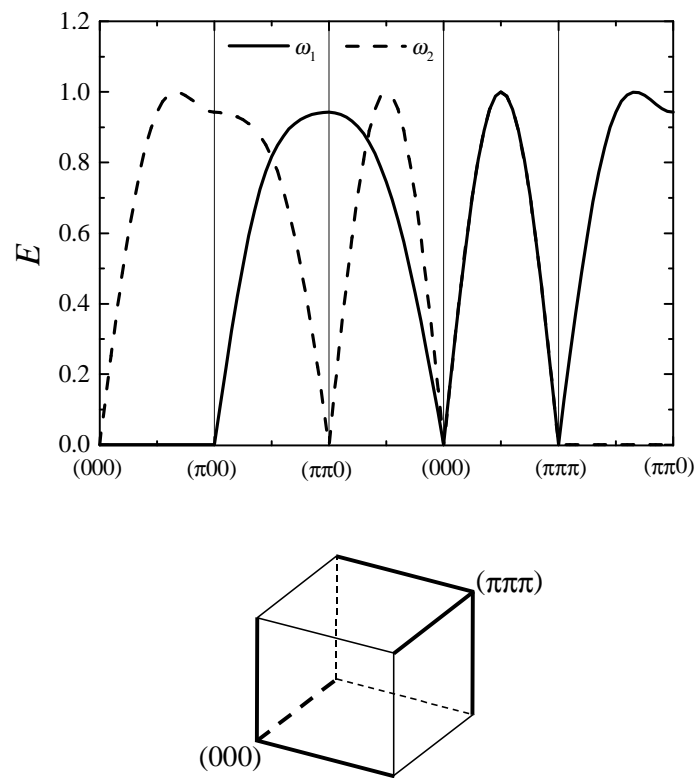

FIG. 5. Upper panel: Orbiton dispersions (in units of $r_{1} J_{S E}$ ), obtained in a linear spin-wave approximation. Lower panel: Positions of soft modes are shown by the thick lines.

$$
\begin{gathered}
T_{ \pm 1}=-\left(\tilde{a}^{\dagger}+\tilde{a}\right) \mp\left(\tilde{b}^{\dagger}+\tilde{b}\right), \\
l_{x}=i\left(\tilde{b}-\tilde{b}^{\dagger}\right), \quad l_{y}=i\left(\tilde{a}^{\dagger}-\tilde{a}\right) .
\end{gathered}
$$

In a momentum space, this linearized Hamiltonian reads as

$$
\begin{aligned}
H_{O W}=\sum_{\vec{k}}[ & n_{\tilde{a} \vec{k}}+n_{\tilde{b} \vec{k}}+\frac{1}{2}\left(\gamma_{1}+\gamma_{2}\right)\left(\tilde{a}_{\vec{k}}^{\dagger} \tilde{a}_{-\vec{k}}^{\dagger}+\tilde{a}_{\vec{k}} \tilde{a}_{-\vec{k}}\right) \\
& +\frac{1}{2}\left(\gamma_{1}-\gamma_{2}\right)\left(\tilde{b}_{\vec{k}}^{\dagger} \tilde{b}_{-\vec{k}}^{\dagger}+\tilde{b}_{\vec{k}} \tilde{b}_{-\vec{k}}\right) \\
& \left.-\gamma_{3}\left(\tilde{a}_{\vec{k}}^{\dagger} \tilde{b}_{-\vec{k}}^{\dagger}+\tilde{a}_{\vec{k}} \tilde{b}_{-\vec{k}}\right)\right]
\end{aligned}
$$

where $\gamma_{1}, \gamma_{2}$, and $\gamma_{3}$ are defined as $\gamma_{1}=\left(c_{x}+c_{y}+c_{z}\right) / 3$, $\gamma_{2}=\sqrt{3}\left(c_{y}-c_{x}\right) / 6$, and $\gamma_{3}=\left(2 c_{z}-c_{x}-c_{y}\right) / 6$, respectively, with $c_{\alpha}=\cos k_{\alpha}$. The Hamiltonian is diagonalized by using Bogoliubov transformation (see for details Appendix B). One obtains:

$$
H_{O W}=\sum_{\vec{k}}\left(\omega_{1 \vec{k}} \alpha_{1 \vec{k}}^{\dagger} \alpha_{1 \vec{k}}+\omega_{2 \vec{k}} \alpha_{2 \vec{k}}^{\dagger} \alpha_{2 \vec{k}}\right)+E_{0}
$$

where $\omega_{1 \vec{k}}=\sqrt{1-\left(\gamma_{1}+\kappa\right)^{2}}$ and $\omega_{2 \vec{k}}=\sqrt{1-\left(\gamma_{1}-\kappa\right)^{2}}$ with $\kappa=\sqrt{\gamma_{2}^{2}+\gamma_{3}^{2}}$. The dispersion relations of orbitons are presented in Fig. 5. Orbital excitations are characterized by the flat dispersion with zero energy along $\left(0,0, k_{z}\right),\left(\pi, \pi, k_{z}\right)$ and their equivalent directions. As discussed later, interaction effects open the gap along $\left(\pi, \pi, k_{z}\right)$ and equivalent ones, while zero modes along $\left(k_{x}, 0,0\right),\left(0, k_{y}, 0\right)$, and $\left(0,0, k_{z}\right)$ are protected by the underlying symmetry of the model.

A constant $E_{0}$ in Eq. (29) represents the energy gain due to the quantum fluctuations. It is given by

$$
E_{0}=\frac{1}{2} \sum_{\vec{k}}\left(\omega_{1 \vec{k}}+\omega_{2 \vec{k}}\right)-1=-0.214\left(r_{1} J_{S E}\right) .
$$

We may compare this result with ground-state energy of the orbital disordered AF state: $E_{0}=$ $-0.33 \frac{r_{1}+r_{2}}{2} J_{S E},{ }^{4}$ where the result of Ref. 4 is corrected for the finite values of $\eta$. For realistic values of the Hund's coupling, say $\eta=J_{H} / U=0.12$, this gives $E_{0}=-0.285$ (in units of $r_{1} J_{S E}$ ) in AF state. It is noticed that ferromagnetic and AF states are almost degenerate. Still, the ferromagnetic state is higher than the AF state, so its stabilization in $\mathrm{YTiO}_{3}$ requires an additional effects as discussed in Sec. IV.

Due to the flat mode, one may expect strong orbital fluctuations in the ground state. Indeed, number of the excited bosons $\tilde{a}$ and $\tilde{b}$ is large even at $T=0$ :

$$
\left\langle n_{i \tilde{a}}+n_{i \tilde{b}}\right\rangle=-1+\frac{1}{2} \sum_{\vec{k}}\left(\frac{1}{\omega_{1 \vec{k}}}+\frac{1}{\omega_{2 \vec{k}}}\right)=0.54
$$

This reduces the condensate density to $\left\langle n_{i \tilde{c}}\right\rangle=0.46$. Consequently, the quadrupole order parameter is obtained to be rather small: $Q=0.19$. Reduction of quadrupole order $Q$ implies that electron density is much less anisotropic than that shown for the classical state in Fig. 4(a). Including fluctuation effects, that is, finite population of $\tilde{a}$ and $\tilde{b}$ orbitals, electron density at site 1 is given by $\rho_{1}(\vec{r})=n_{\tilde{c}} \psi_{\tilde{c}}^{2}+n_{\tilde{a}} \psi_{\tilde{a}}^{2}+n_{\tilde{b}} \psi_{\tilde{b}}^{2}$. Using Eq. (21), one then finds

$$
\begin{aligned}
\rho_{1}(\vec{r})= & \frac{1}{3}\left(d_{y z}^{2}+d_{x z}^{2}+d_{x y}^{2}\right) \\
& +\frac{2}{3} Q\left(d_{y z} d_{x z}+d_{y z} d_{x y}+d_{x z} d_{x y}\right) .
\end{aligned}
$$

Electron density at other sites is given by a similar equation, where the second, of $t_{2 g}$ symmetry term, is different for different sublattices. Namely, it is $\left(d_{y z} d_{x z}-d_{y z} d_{x y}-\right.$ $\left.d_{x z} d_{x y}\right)$ for site $2,\left(-d_{y z} d_{x z}+d_{y z} d_{x y}-d_{x z} d_{x y}\right)$ for site 3 , and $\left(-d_{y z} d_{x z}-d_{y z} d_{x y}+d_{x z} d_{x y}\right)$ for site 4 . In Fig. 6(b), we present the electron distribution given by Eq. (32). For comparison, we show in Fig. 6(a) the electron distribution where $\tilde{a}, \tilde{b}$, and $\tilde{c}$ are equally occupied. At finite $Q$, the electron density $\rho_{1}(\vec{r})$ is slightly elongated along [111] direction. Thus, we expect the quadrupole ordered state to be further stabilized by the electron lattice coupling, although this coupling is expected to be weak for $t_{2 g}$ orbitals.

The anomalous reduction of the order parameter is due to the highly frustrated nature of the interactions in Eq. (14). A special, non-spin-like feature of all orbital models is that orbitals are bond selective, resulting in a pathological degeneracy of classical states. This leads to soft modes [observe that $\omega_{1,2 \vec{k}}$ is just flat along $(0,0, \pi)$ and equivalent directions]. These soft modes have their origin in special symmetry properties of the $t_{2 g}$ orbital model Eq. (14), which result in conservation laws with important consequences. Namely, the total number of 


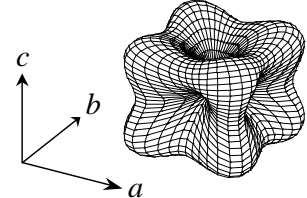

(a)

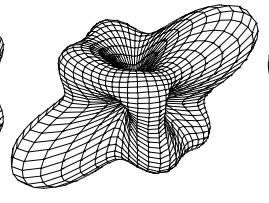

(b)

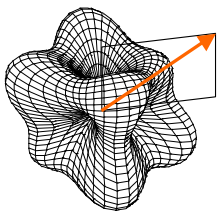

(c)
FIG. 6. (Color online). Spatial electron distribution in different states. (a)Disordered state, $n_{a}=n_{b}=n_{c}=\frac{1}{3}$. (b)Quadrupole ordered state, $n_{\tilde{c}}=0.46$ and $n_{\tilde{a}}=n_{\tilde{b}}=0.27$. (c)Orbital magnetic state, $n_{\bar{a}}=0.46$ and $n_{\bar{b}}=n_{\tilde{c}}=0.27$. Arrow shows the direction of angular momentum.

orbitals of each "color" $(a, b, c)$ are conserved during superexchange process, as can easily be seen from Eq.(4). Moreover, as $t_{2 g}$-orbitals can hop only along two directions [say, $x y$-orbital motion is restricted to $(a b)$ planes], the orbital number is conserved on each plane separately. Formally, these conservation rules are reflected by a possibility of uniform phase transformation of orbiton operators, e.g., $a \rightarrow a \exp \left(i \phi_{a}\right)$, etc., which leaves the orbital Hamiltonian invariant. These continuous symmetries are spontaneously broken in above ordered states. The breaking of continuous symmetry is usually followed by the generation of gapless Goldstone modes. This is precisely what happens in the $t_{2 g}$ orbitally degenerate model. In fact, soft modes obtained above have twodimensional (2D) feature (stemming from 2D geometry of the $t_{2 g}$ orbital hoppings). As a result, long range orbital order is possible only at zero temperature, just like in 2D Heisenberg models. Formally, this is manifested as a divergence ( $\operatorname{as} \ln \frac{1}{T}$ ) of the number of thermally excited orbitons, $\left\langle n_{\tilde{a}}+n_{\tilde{b}}\right\rangle$, if one attempts to calculate this quantity at finite temperature, including the Bose population factor in Eq.(31). Soft modes will be discussed in more detail later on.

\section{Orbital magnetic order}

In order to describe the magnetic ordering (denoted above by state II), let us introduce orbital states

$$
\bar{a}=\frac{1}{\sqrt{2}}(\tilde{a}-i \tilde{b}), \quad \bar{b}=\frac{1}{\sqrt{2}}(\tilde{a}+i \tilde{b}) .
$$

In these states, angular momentum has eigenvalues $\tilde{l}_{z}=$ \pm 1 (on local axes), respectively. A condensation of $\bar{a}$ leads therefore to the magnetic ordering (with $\tilde{l}_{z}=1$ classically), and it is associated with ordering of the complex orbital:

$$
\psi_{l}=\frac{1}{\sqrt{3}}\left\{d_{y z} e^{i \frac{\pi}{3}}+d_{x z} e^{-i \frac{\pi}{3}}-d_{x y}\right\} .
$$

On global axes, this order is noncollinear, as shown in Fig. 4(b). We consider fluctuations of this state. Using the relation $\bar{a}=\bar{a}^{\dagger}=\sqrt{1-n_{\bar{b}}-n_{\tilde{c}}}$ and expanding interactions in Eq. (23) up to second order in $\bar{b}$ and $\tilde{c}$, we obtain the following linearized Hamiltonian:

$$
\begin{aligned}
H_{O W}=\sum_{i}\left(n_{i \bar{b}}+\right. & \left.n_{i \tilde{c}}\right) \\
+\frac{1}{2 z} \sum_{\langle i j\rangle}[ & Q_{i x} T_{j-1}+T_{i-1} Q_{j x}-T_{i 0} T_{i 1}-T_{i 1} T_{j 0} \\
& +Q_{i x}^{(\gamma)} Q_{j x}^{(\gamma)}-T_{i 0}^{(\gamma)} T_{j 0}^{(\gamma)}+T_{i-1}^{(\gamma)} T_{j-1}^{(\gamma)} \\
& \left.\quad\left(l_{x}+l_{y}\right)_{i}^{(\gamma)}\left(l_{x}+l_{y}\right)_{j}^{(\gamma)}\right] .
\end{aligned}
$$

Here, the operators $l, Q$, and $T$ are linear functions of $\bar{b}$ and $\tilde{c}$ :

$$
\begin{gathered}
l_{x}=-\frac{1}{\sqrt{2}}\left(\tilde{c}^{\dagger}+\tilde{c}\right), \quad l_{y}=-\frac{i}{\sqrt{2}}\left(\tilde{c}^{\dagger}-\tilde{c}\right), \\
Q_{x}=-\left(\bar{b}^{\dagger}+\bar{b}\right), \quad T_{0}=-i\left(\bar{b}^{\dagger}-\bar{b}\right), \\
T_{ \pm 1}=-\frac{1}{\sqrt{2}}\left[(1 \mp i) \tilde{c}+(1 \pm i) \tilde{c}^{\dagger}\right] .
\end{gathered}
$$

We introduce now new operators $\alpha, \beta$ :

$$
\alpha=\frac{1}{\sqrt{2}}\left(\bar{b}+e^{i \varphi} \tilde{c}\right), \quad \beta=\frac{1}{i \sqrt{2}}\left(\bar{b}-e^{i \varphi} \tilde{c}\right),
$$

with $\varphi=\pi / 4$. Remarkably, after this transformation the linearized Hamiltonian obtains in a momentum space the same form as Eq. (28), where $\tilde{a}, \tilde{b}$ are just replaced by $\alpha, \beta$, and $\gamma_{2}$ and $\gamma_{3}$ are interchanged. Exchange of $\gamma_{2}$ and $\gamma_{3}$ does not affect the excitation spectrum, however, as they enter in $\omega_{1,2 \vec{k}}$ via the parameter $\kappa=\sqrt{\gamma_{2}^{2}+\gamma_{3}^{2}}$ only. Thus, the ground-state energy in the orbital magnetic state $E_{0}$ is given again by Eq. (30), so the states I and II are degenerate even on quantum level. Similarly, the number of out of condensate bosons is also obtained from Eq. (31). This gives $\left\langle n_{\alpha}+n_{\beta}\right\rangle=0.54$, and values for the angular and quadrupole momentum order parameters follow:

$$
\begin{aligned}
m_{l} & =1-\frac{3}{2}\left\langle n_{\alpha}+n_{\beta}\right\rangle=0.19, \\
Q & =-\frac{1}{2}+\frac{3}{4}\left\langle n_{\alpha}+n_{\beta}\right\rangle=-0.095 .
\end{aligned}
$$

Electron-density distribution in the state II, given by Eq. (32) with above value of $Q$, is shown in Fig. 6 (c). Electron cloud in the magnetic state is almost of cubic symmetry, being just slightly contracted along [111] direction (opposite to that in the state I). Thus the energy gain from the orbital lattice coupling is smaller in this state. In principle, orbital magnetic order could be supported by a relativistic spin-orbital coupling; however, in the noncollinear state driven by the superexchange interaction, uniform component of the orbital moment is zero [see Fig. 4(b)], hence the coupling to the spin ferromagnetism vanishes in linear order. The spin-orbital energy gain in a second order is possible though, via the canting of spins towards orbital magnetic pattern. 


\section{Soft modes: density-phase formulation}

Having obtained an identical excitation spectrum for states with apparently different ground-state condensates, we would like to unify these states. It is convenient to use a different approach, that is, the densityphase formulation by Popov (Ref. 20), nowadays called "radial gauge". This formalism is particularly useful also to clarify the physical origin of the soft modes obtained above. In the radial gauge, density and phase degrees of freedom of the constrained particles are emphasized. We work in a basis obtained by first transformation, Eq.(15), and represent the orbiton operators entering in Eq.(16) as follows:

$$
\alpha_{i}=\sqrt{\rho_{i \alpha}} e^{i \theta_{i \alpha}} \quad(\alpha=a, b, c) .
$$

Further, the density and phase degrees of freedom are parametrized as

$$
\begin{aligned}
\rho_{i a(b)} & =\rho_{0}+\frac{2}{3}\left(c r_{i} \pm s \lambda_{i}\right), \\
\rho_{i c} & =\rho_{0}-\frac{2}{3} r_{i}
\end{aligned}
$$

and

$$
\begin{aligned}
\theta_{i a(b)} & =\Omega_{i}+c \varphi_{i} \pm s \theta_{i}, \\
\theta_{i c} & =\Omega_{i}-\varphi_{i},
\end{aligned}
$$

respectively. Here, $\rho_{0}(=1 / 3)$ is an average electron density on each orbital. The phase $\Omega_{i}$, common to all the three orbitons, can as usually be absorbed by the constraint field, while the local constraint itself is explicitly resolved by parametrization (40). The physically active degrees of freedom are therefore $r$ and $\lambda$ fields for the amplitude fluctuations, and $\theta$ and $\varphi$ for the phase fluctuations. We recall that the coefficients $c=1 / 2, s=\sqrt{3} / 2$.

To start with, let us neglect for a moment the amplitude fluctuations, and focus on the phase dependence of the classical condensate wave function. In terms of phases in Eq.(41), it is written as follows (up to unessential overall phase factor):

$\psi(\theta, \varphi)=\sqrt{\rho_{0}}\left\{d_{y z} e^{i(3 c \varphi+s \theta)}+d_{x z} e^{i(3 c \varphi-s \theta)}+d_{x y}\right\}$.

Here, we suppressed site dependence of the phases $\varphi, \theta$, discarding for a while slow space variations of the condensate. Now, it is noticed that the quadrupole and magnetic orderings [see Eqs.(21) and (34)] do follow from Eq.(42) when $\varphi=\theta=0$, and $\varphi=\frac{\pi}{3 c}, \theta=\frac{\pi}{3 s}$, respectively. Next observation is the orbital "color" conservation rule in the ferromagnetic state. In the radial gauge, it is evident from Eq.(16), that the interactions do depend on the difference of the orbiton phases only, that is on $\theta_{i a}-\theta_{j a}$, etc., so we can uniformly rotate the condensate function (42) by arbitrary phases $\varphi, \theta$ with no energy cost. By such rotations, we can in fact mix quadrupole (state I) and magnetic (state II) orderings. Slow phase rotation of the condensate is precisely the origin of the soft modes obtained above. Because of the two dimensionality of $t_{2 g}$ orbitals, the phases can spontaneously be fixed at zero temperature only. Of course, orbital-lattice and/or spin-orbital couplings may fix the phases, thus selecting a particular state even at finite $T$.

We now turn to the excitations of the model in densityphase formulation. $A_{i j}^{(\gamma)}$ in Eq. (16) is expressed as

$$
\begin{aligned}
A_{i j}^{(c)} & =\left(\sqrt{\rho_{i a} \rho_{j a}}-\sqrt{\rho_{i b} \rho_{j b}}\right)^{2} \\
& +2 \sqrt{\rho_{i a} \rho_{j a}} \sqrt{\rho_{i b} \rho_{j b}}\left\{1-\cos \left(\phi_{i}^{(c)}-\phi_{j}^{(c)}\right)\right\},
\end{aligned}
$$

where $\phi_{i}^{(c)}=\theta_{i a}-\theta_{i b}$. $A_{i j}^{(\gamma)}$ for $\gamma=a$ and $b$ bonds are given by replacing $(a, b)$ in Eq. (43) with $(b, c)$ and $(c, a)$, respectively. In terms of the relevant phase degrees of freedom $\varphi$ and $\theta$, we obtain

$$
\phi_{i}^{(\gamma)}=\left\{\begin{aligned}
\sqrt{3}\left(-c \theta_{i} \pm s \varphi_{i}\right), & \text { for } \gamma=a(b) \\
\sqrt{3} \theta_{i} . & \text { for } \gamma=c
\end{aligned}\right.
$$

The density operators $\rho_{i \alpha}$ are functionals of the $r_{i}$ and $\lambda_{i}$ fields. We may expand now the operator $A_{i j}^{(\gamma)}$ in terms of the amplitude and phase variables $r, \lambda, \varphi, \theta$. Keeping quadratic only terms in the expansion, one arrives at the following linearized Lagrangian for the phase-amplitude fluctuations

$L_{o r b}=\sum_{i, w} w\left(\theta_{i, w} \lambda_{i,-w}+\varphi_{i, w} r_{i,-w}\right)+H_{\theta \varphi}+H_{\lambda r}$,

where $\omega$ is the Matsubara frequency. The first term in this equation originates from the time derivative, kinematic part of the Lagrangian $-\sum_{i, \alpha} \alpha_{i}^{\dagger} \frac{\partial}{\partial \tau} \alpha_{i}$, and produces dynamical coupling between the density and phase variables. $H_{\theta \varphi}$ represents the phase fluctuations, and it is obtained from the expansion of the second term in Eq. (43):

$$
H_{\theta \varphi}=\frac{1}{2} \rho_{0}^{2} \sum_{\langle i j\rangle}\left(\phi_{i}^{(\gamma)}-\phi_{j}^{(\gamma)}\right)^{2}
$$

The fluctuations of the condensate density about $\rho_{0}$ are controlled by the Hamiltonian $H_{\lambda r}$, which in a linear approximation reads as

$$
H_{\lambda r}=\frac{9}{8} \rho_{0}^{2} \sum_{\langle i j\rangle}\left(\delta_{i}^{(\gamma)}+\delta_{j}^{(\gamma)}\right)^{2}
$$

Here, $\delta_{i}^{(\gamma)}$ is the difference between the electron densities on orbitals that are active on a given direction: $\delta_{i}^{(\gamma)}=\rho_{i \alpha}^{(\gamma)}-\rho_{i \beta}^{(\gamma)}$. Using parametrization (40), they are expressed via $r$ and $\lambda$ fields as follows:

$$
\delta_{i}^{(\gamma)}=\left\{\begin{aligned}
\frac{2}{\sqrt{3}}\left(-c \lambda_{i} \pm s r_{i}\right), & & \text { for } \gamma=a(b) \\
\frac{2}{\sqrt{3}} \lambda_{i} . & & \text { for } \gamma=c
\end{aligned}\right.
$$


In a momentum space, $H_{\theta \varphi}$ and $H_{\lambda r}$ are represented as follows:

$$
\begin{aligned}
& H_{\theta \varphi}=\sum_{\vec{q}}\left\{\frac{1}{2} a_{\theta}\left|\theta_{\vec{q}}\right|^{2}+\frac{1}{2} a_{\varphi}\left|\varphi_{\vec{q}}\right|^{2}-\gamma_{2} \varphi_{\vec{q}} \theta_{-\vec{q}}\right\}, \\
& H_{\lambda r}=\sum_{\vec{q}}\left\{\frac{1}{2} a_{\lambda}\left|\lambda_{\vec{q}}\right|^{2}+\frac{1}{2} a_{r}\left|r_{\vec{q}}\right|^{2}+\gamma_{2} \lambda_{\vec{q}} r_{-\vec{q}}\right\},
\end{aligned}
$$

where $a_{\theta}=1-\left(\gamma_{1}+\gamma_{3}\right), a_{\varphi}=1-\left(\gamma_{1}-\gamma_{3}\right), a_{\lambda}=$ $1+\left(\gamma_{1}+\gamma_{3}\right)$, and $a_{r}=1+\left(\gamma_{1}-\gamma_{3}\right)$.

Equations (45) and (49), (50) determine the orbital dynamics in a harmonic approximation, which is equivalent to the previous linear orbital wave approach. Indeed, the obtained quadratic form in Eq.(45) can easily be diagonalized giving exactly the same excitation spectrum, that is $\sqrt{1-\left(\gamma_{1} \pm \kappa\right)^{2}}$ found in the preceding section. In addition, the origin of zero energy excitations can clearly be identified now. In a classical limit [neglect dynamical term in Eq.(45)], the quadratic forms $H_{\theta \varphi}$ (49) and $H_{\lambda r}$ (50) can be diagonalized separately resulting in normal modes with energies $\omega_{\theta}^{ \pm}(\vec{k})=\left(1-\gamma_{1} \pm \kappa\right)$ in phase sector, and $\omega_{\rho}^{ \pm}(\vec{k})=\left(1+\gamma_{1} \pm \kappa\right)$ for the amplitude variables. $\omega_{\theta}^{ \pm}$vanishes on lines $\left(k_{x}, 0,0\right),\left(0, k_{y}, 0\right),\left(0,0, k_{z}\right)$ (see Fig. 5). Therefore, zero-energy excitations on these lines do correspond to uniform phase rotations of orbitons on different planes as discussed before. On the other hand, the normal modes $\omega_{\rho}^{ \pm}(\vec{k})$ that are associated with the density of orbital occupancies possess zero lines at $\left(k_{x}, \pi, \pi\right),\left(\pi, k_{y}, \pi\right),\left(\pi, \pi, k_{z}\right)$. This reflects softness of the staggered fluctuations of orbitals [notice also that a uniform component, that is, $\delta_{u}^{(\gamma)}=\delta_{i}^{(\gamma)}+\delta_{j}^{(\gamma)}$, only enters in Eq.(47)]. However, such soft modes in the amplitude sector are not protected by physical conservation rules; therefore, they are expected to acquire a finite mass due to interaction effects that go beyond linear orbital wave approximation. To see this, one should consider unharmonic terms in the expansion of Eq.(43). Most relevant term in that expansion is the interaction between the staggered fluctuations, that is, $\delta_{s}^{(\gamma)}=\delta_{i}^{(\gamma)}-\delta_{j}^{(\gamma)}$, with uniform components $\delta_{u}^{(\gamma)}$. When such a term is kept, Eq.(47) is replaced by

$$
\begin{aligned}
H_{\lambda r} & =\frac{9}{8} \rho_{0}^{2} \sum_{\langle i j\rangle}\left\{\delta_{u}^{2}+\frac{1}{\left(4 \rho_{0}\right)^{2}} \delta_{s}^{2} \delta_{u}^{2}\right\}^{(\gamma)} \\
& \simeq \frac{9}{8} \rho_{0}^{2} \sum_{\langle i j\rangle}\left\{\left(1+\varepsilon_{s}\right) \delta_{u}^{2}+\varepsilon_{u} \delta_{s}^{2}\right\}^{(\gamma)} .
\end{aligned}
$$

The Hartree decoupling is applied here to the interaction term, with $\varepsilon_{s, u}=\left(1 / 4 \rho_{0}\right)^{2}\left\langle\delta_{s, u}^{2}\right\rangle$. These expectation values are finite due to the presence of quantum fluctuations in the ground state that are particularly enhanced in a staggered channel. In a momentum space, the above equation reads as

$$
H_{\lambda r}=Z_{\varepsilon} \sum_{\vec{q}}\left\{\frac{1}{2} \tilde{a}_{\lambda}\left|\lambda_{\vec{q}}\right|^{2}+\frac{1}{2} \tilde{a}_{r}\left|r_{\vec{q}}\right|^{2}+\gamma_{2}^{\varepsilon} \lambda_{\vec{q}} r_{-\vec{q}}\right\}
$$

where $\tilde{a}_{\lambda}=1+\left(\gamma_{1}^{\varepsilon}+\gamma_{3}^{\varepsilon}\right)$ and $\tilde{a}_{r}=1+\left(\gamma_{1}^{\varepsilon}-\gamma_{3}^{\varepsilon}\right) \cdot \gamma_{n}^{\varepsilon}(n=$ $1,2,3)$ are defined as $\gamma_{n}^{\varepsilon}=(1-2 \varepsilon) \gamma_{n}$ with $\varepsilon=\varepsilon_{u} / Z_{\varepsilon}$, and $Z_{\varepsilon}=\left(1+\varepsilon_{u}+\varepsilon_{s}\right)$ is an overall rescaling factor.

Using now $H_{\lambda r}$ given by Eq. (52) in $L_{\text {orb }}$ [Eq. (45)], one obtains finally the following two eigenfrequencies:

$$
\omega_{ \pm}(\vec{k})=\left\{1-(1-2 \varepsilon)\left(\gamma_{1} \pm \kappa\right)^{2}-2 \varepsilon\left(\gamma_{1} \pm \kappa\right)\right\}^{1 / 2}
$$

in units of $\sqrt{Z_{\varepsilon}} r_{1} J_{S E}$. It should be noted that $\omega_{ \pm}$recover the orbiton energy $\omega_{1,2}$ when $\varepsilon=0$. Using bare orbiton dispersions, the Hartree decoupling parameters $\varepsilon_{s, u}$ are calculated as follows:

$$
\begin{aligned}
& \varepsilon_{s}=\frac{3}{4} \sum_{\vec{k}}\{\left.\left(1+\gamma_{1}^{2}+\kappa^{2}\right)+\frac{1-\left(\gamma_{1}^{2}-\kappa^{2}\right)^{2}}{\omega_{1 \vec{k}} \omega_{2 \vec{k}}}\right\} \\
& \times \frac{1}{\omega_{1 \vec{k}}+\omega_{2 \vec{k}}}, \\
& \varepsilon_{u}=\frac{3}{4} \sum_{\vec{k}}\left\{\left(1-\gamma_{1}^{2}-\kappa^{2}\right)+\omega_{1 \vec{k}} \omega_{2 \vec{k}}\right\} \frac{1}{\omega_{1 \vec{k}}+\omega_{2 \vec{k}}} .
\end{aligned}
$$

Numerical calculation gives $\varepsilon_{s}=1.72$ and $\varepsilon_{u}=0.59$, reflecting that staggered fluctuations of densities are stronger. Thus we obtain $\sqrt{Z_{\varepsilon}}=1.82$ and $\varepsilon \simeq 0.18$. Dispersion relations of the orbital excitations, Eq.(53) calculated using these parameters are presented in Fig. 7 (in units of $\sqrt{Z_{\varepsilon}} r_{1} J_{S E}$ ). Staggered density fluctuations are gapped, and we are left now with true Goldstone phase modes protected by the symmetry of interactions.

\section{WHY $\mathrm{YTiO}_{3}$ HAS A FERROMAGNETIC GROUND STATE}

So far, we discussed $t_{2 g}$ orbital physics on an ideal cubic lattice assuming a spin saturated ferromagnetic state. In the remainder of the paper, we apply the theory to the ferromagnetic state of Mott insulator $\mathrm{YTiO}_{3}$. This requires some modifications of the theory implementing a specific feature of this material. On empirical grounds, it is well documented that Ti-O-Ti bond angle is an important parameter controlling magnetic properties of titanates $R \mathrm{TiO}_{3}{ }^{8}$ The bond angle $\theta$ gradually decreases from $\sim 157$ deg in $\mathrm{LaTiO}_{3}$ to $\sim 142$ deg in $\mathrm{YTiO}_{3}$, due to lanthanum contraction effect that results in deviations of the lattice from an ideal perovskite structure. It is quite remarkable that such a small variation of the bond angle, driven by $R$-ionic size effect, affects the magnetic state dramatically: It changes from isotropic $\mathrm{AF}$ as observed in $\mathrm{LaTiO}_{3}$ to the isotropic ferromagnetic state in a Ybased compound, indicating strong competition between $\mathrm{AF}$ and $\mathrm{F}$ interactions in titanates.

Ti-O-Ti bond distortion is important because it induces an unfrustrated ferromagnetic interaction, changing thereby a delicate balance between AF and Ferro couplings that dynamically coexist and compete in ideal $t_{2 g}$ 

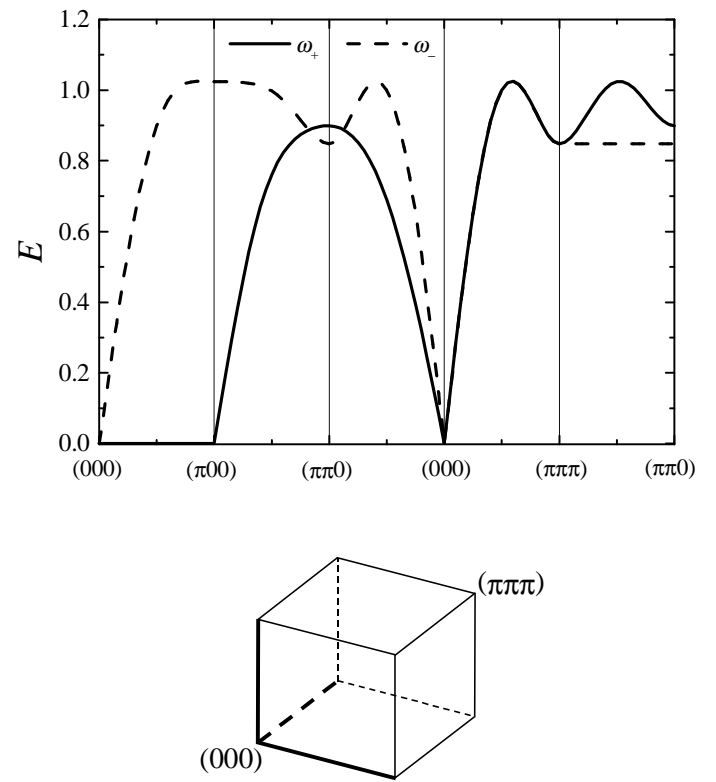

FIG. 7. Orbiton dispersions corrected by interaction effects [Eq. (53)]. Energy is given in units of $Z_{\varepsilon}^{1 / 2} r_{1} J_{S E} \simeq 1.82 r_{1} J_{S E}$. The amplitude fluctuations open the excitation gap around $(\pi \pi \pi)$, while there still remain gapless Goldstone modes at positions, indicated by the thick lines in the lower panel.

superexchange models like in Eq.(13). It was found in Sec. III B that the ferromagnetic state is slightly higher in energy that $G$-type AF one; the situation is however reversed when the bond angle is reduced below some critical value, as we argue below.

The bond distortion brings about the following two effects. (i) Reduction of transfer intensity between nearstneighbor (NN) $t_{2 g}$ orbitals as $t=\Delta_{d p}^{-1} t_{d p \pi}^{2} \cos \theta=$ $t_{0} \cos \theta .^{8}$ Here, $t_{d p \pi}\left(t_{d p \sigma}\right)$ is the transfer between Ti $3 d$ and $\mathrm{O} 2 p$ orbitals on the $\pi(\sigma)$ bond, and $\Delta_{d p}$ is the level difference between Ti $3 d$ and O $2 p$ states. Superexchange energy scale is then reduced as $J_{S E}=J_{S E}^{(0)} \cos ^{2} \theta$ with $J_{S E}^{(0)}=4 t_{0}^{2} / U$. (ii) Generation of transfer intensity between NN $t_{2 g}$ and $e_{g}$ orbitals, $t^{\prime}=\Delta_{d p}^{-1} t_{d p \sigma} t_{d p \pi} \sin \theta$. This transfer induces an additional SE interaction. For $c$-bond, we consider the following transfer term:

$$
H_{t}^{\prime(c)}=t^{\prime}\left[\left(\alpha_{i \sigma}^{\dagger} a_{j \sigma}+h . c .\right)+(i \leftrightarrow j)\right] .
$$

$\alpha^{\dagger}$ denotes the creation operator of electron in the $e_{g}$ orbital with $3 z^{2}-r^{2}$ symmetry. $H^{\prime}{ }_{t}$ for $a$ - and $b$-bonds are given by replacing $(\alpha, a)$ in Eq. (56) by $[-(\alpha-\sqrt{3} \beta) / 2, b]$ and $[-(\alpha+\sqrt{3} \beta) / 2, c]$, respectively, where $\beta$ denotes the electron annihilation operator in the $e_{g}$ orbital with $x^{2}-y^{2}$ symmetry. By the second-order perturbation with respect to $H_{t}^{\prime}$, one obtains the SE interaction between NN $t_{2 g}$ electrons. Here, energy of the intermediate $d^{2}$ excited states with spin triplet and singlet states between $e_{g}$ and $t_{2 g}$ electrons is given by $U-3 J_{H}+\Delta_{c r}$ and $U-J_{H}+\Delta_{c r}$, respectively, where $\Delta_{c r}$ is a cubic crystal-field splitting between $e_{g}$ and $t_{2 g}$ levels (so-called 10Dq). Explicit expression for the new $\mathrm{SE}$ interaction is

$$
\begin{aligned}
{H_{S E}^{\prime(c)}}_{S}= & -\frac{1}{8} J_{S E}\left(\frac{t^{\prime}}{t}\right)^{2} \frac{U}{\widetilde{U}} \frac{1}{(1-3 \tilde{\eta})(1-\tilde{\eta})} \\
& \times\left(2-3 \tilde{\eta}+4 \tilde{\eta} \vec{S}_{i} \cdot \vec{S}_{j}\right)\left(n_{i a}+n_{j a}\right),
\end{aligned}
$$

where $\widetilde{U}=U+\Delta_{c r}$ and $\tilde{\eta}=J_{H} / \widetilde{U} . \quad H^{\prime}{ }_{S E}$ for $a(b)$ bonds are given by replacing $n_{a}$ in Eq. (57) by $n_{b}\left(n_{c}\right)$. It is stressed that this SE interaction is of the ferromagnetic sign, because $t_{2 g}$ and $e_{g}$ orbitals are of the different symmetry, and the Hund coupling between them favors spin triplet state. As Ti-O-Ti bond angles in $a, b$, and $c$ directions are almost the same, ${ }^{8}$ this interaction supports ferromagnetism equally in all three directions. Here we differ from Ref. 21, which considers $t_{2 g}-e_{g}$ hopping channel along the $c$-axis only.

Either in the orbital-ordered states [I (a) and I (b)] with $\psi_{\tilde{c}}=\frac{1}{\sqrt{3}}\left(d_{y z}+d_{x z}+d_{x y}\right)$, or in the orbital-liquid one, average occupation number of each $t_{2 g}$ orbital is given by $n_{a}=n_{b}=n_{c}=1 / 3$. Thus, the spin interaction (57) in these orbital states becomes $H_{\text {spin }}^{\prime}=-J^{\prime} \sum_{\langle i j\rangle} \vec{S}_{i} \cdot \vec{S}_{j}$ with

$$
J^{\prime}=\frac{1}{3} J_{S E}\left(\frac{t^{\prime}}{t}\right)^{2}\left(\frac{U}{\widetilde{U}}\right)^{2} \eta .
$$

One should notice that $J^{\prime}$ is proportional to $\sin ^{2} \theta$ (via $t^{\prime}$ ), and contains also the small number $\eta=J_{H} / U$. This is because $J^{\prime}$ is caused by the Hund coupling between $t_{2 g}$ and $e_{g}$ electrons in the virtually excited $d^{2}\left(e_{g}^{1} t_{2 g}^{1}\right)$ state, which is evoked in SE process only in the presence of Ti-O-Ti bond angle distortion.

The energy difference between $\mathrm{AF}$ and ferromagnetic phases stemming from $H_{\text {spin }}^{\prime}$ is given by $\Delta E_{S E}^{\prime}=\frac{1}{2} J^{\prime}$, while that from $H_{S E}$ is given by $\Delta E_{S E}=\left(-0.33 \frac{r_{1}+r_{2}}{2}+\right.$ $\left.0.214 r_{1}\right) J_{S E}$ (Sec. III B). The total SE energy difference between $\mathrm{AF}$ and ferromagnetic phases is then estimated as

$\Delta E=\left[-0.33 \frac{r_{1}+r_{2}}{2}+0.214 r_{1}+\frac{1}{2}\left(\frac{t^{\prime}}{t}\right)^{2}\left(\frac{U}{\widetilde{U}}\right)^{2} \eta\right] J_{S E}$.

Thus, $\Delta E$ obtains the following $\theta$ dependence (at representative value $\eta=0.12$ for Hund's coupling parameter):

$$
\Delta E=\left[-0.111 \cos ^{2} \theta+\frac{1}{2}\left(\frac{t_{d p \sigma}}{t_{d p \pi}}\right)^{2}\left(\frac{U}{\widetilde{U}}\right)^{2} \eta \sin ^{2} \theta\right] J_{S E}^{(0)} .
$$

With the realistic parameters (typically, $\Delta_{c r}$ is about 2 eV) $U / \Delta_{c r}=2.5$ and $t_{d p \sigma} / t_{d p \pi}=2$, the transition from $\mathrm{AF}$ orbital-liquid phase to ferromagnetic orbital-ordered one occurs at the critical angle $\theta_{c}^{(0)}=136 \mathrm{deg}$ in the 
(a)

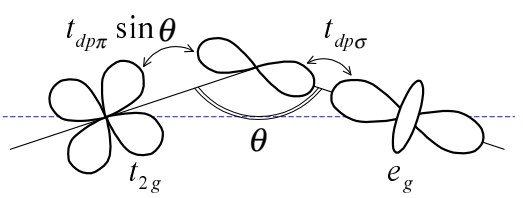

(b)

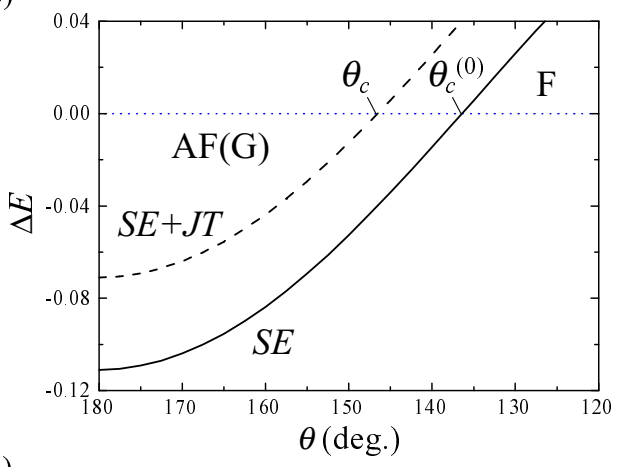

(c)

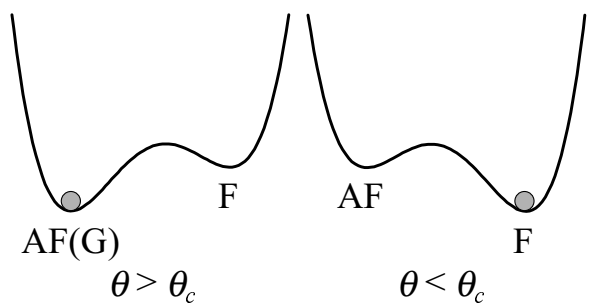

FIG. 8. (a) $t_{2 g}-e_{g}$ transfer originating from Ti-O-Ti bond distortion. Ti-O-Ti bond angle is denoted by $\theta$. (b) Energy difference $\Delta E$ (in units of $J_{S E}^{(0)}$ ) between spin-AF $(\mathrm{G}) /$ orbital-liquid and spin-F/orbital-ordered states as function of $\theta$. The solid line is a result of purely electronic, SE interactions. The broken line includes small JT energy gain $\delta E_{J T}$ in the orbitally ordered ferromagnetic state. Parameters are $\eta=0.12, U / \Delta_{c r}=2.5, t_{d p \sigma} / t_{d p \pi}=2$, and $\delta E_{J T} / J_{S E}^{(0)}=-0.04$. (c) Schematic energy diagrams at $\theta>\theta_{c}$ (left) and at $\theta<\theta_{c}$ (right).

SE model. This angle is slightly smaller than that observed in $\mathrm{YTiO}_{3}(\theta \sim 142 \mathrm{deg})$. Further, orbital-ordered state should be favored over the AF orbital-liquid state by orbital-lattice coupling. We simulate this by adding JT energy gain $\delta E_{J T}(<0)$ to the energy of the ferromagnetic orbital-ordered state. As shown in Fig. 8 (b), this increases $\theta_{c}$. A value of $\delta E_{J T}$, which is required to obtain a realistic value $\theta_{c}=146 \mathrm{deg}$ for titanates is small $\left(-0.04 J_{S E}^{(0)}\right)$, so it might be hard to observe the associated

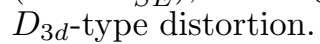

\section{ORBITAL GAP IN $\mathrm{YTIO}_{3}$}

Effect of $\mathrm{TiO}_{6}$ tilting: In addition to the finite transfer between NN $t_{2 g}-e_{g}$ orbitals, octahedron tilting changes also the symmetry of NN $t_{2 g}-t_{2 g}$ hopping matrix, making possible finite electron transfer between the NN orbitals with different symmetry. ${ }^{17}$ We show now that such a hopping leads to an important modification of the or- bital excitation spectrum, removing gapless Goldstone modes. Taking into account nondiagonal hoppings between orbitals active on a given direction

$$
t^{\prime \prime}\left[\left(\alpha_{i}^{\dagger} \beta_{j}+\beta_{i}^{\dagger} \alpha_{j}\right)+(i \leftrightarrow j)\right]^{(\gamma)}
$$

up to second order in small ratio $t^{\prime \prime} / t$, we obtain the following correction to the interaction between NN orbitals in a spin-ferromagnetic state:

$$
\begin{aligned}
H_{S E}^{\prime \prime(\gamma)} & =r_{1} J_{S E}\left(\frac{t^{\prime \prime}}{t}\right)\left[\tau_{i x}^{(\gamma)}\left(1-n_{j}^{(\gamma)}\right)+\tau_{j x}^{(\gamma)}\left(1-n_{i}^{(\gamma)}\right)\right] \\
& +\frac{1}{2} r_{1} J_{S E}\left(\frac{t^{\prime \prime}}{t}\right)^{2}\left[B_{i j}^{(\gamma)}-n_{i}^{(\gamma)} n_{j}^{(\gamma)}\right]
\end{aligned}
$$

Here $B_{i j}^{(\gamma)}$ is given by either of Eqs. (5),(9), and (12). A crucial point is that this operator violates the orbital "color" conservation rule even in the fully spin polarized state. Therefore, uniform phase rotations (separately on each orbital flavor) are not longer possible, hence the relative phases will be fixed and orbital gap will be generated.

Let us apply a radial gauge description, and focus on phase fluctuations, as the amplitude fluctuations have a large gap anyhow. In a local coordinates [defined by Eq.(15)], we find

$$
H_{\theta \varphi}^{\prime \prime(\gamma)}=\frac{1}{2} r_{1} J_{S E} \rho_{0}^{2}\left(\frac{t^{\prime \prime}}{t}\right)^{2} \sum_{\langle i j\rangle}\left(\phi_{i}^{(\gamma)}+\phi_{j}^{(\gamma)}\right)^{2}
$$

Here, we expanded the function $\cos \left(\phi_{i}^{(\gamma)}+\phi_{j}^{(\gamma)}\right)$, which enters in $B_{i j}^{(\gamma)}$, about $\theta, \varphi=0$ (this corresponds to the quadrupole ordered state, which is in fact favored by $B_{i j}^{(\gamma)}$ term).

Effect of trigonal $\mathrm{TiO}_{6}$ distortion: Our orbital state would be supported also by a trigonal $\left(D_{3 d}\right)$ distortion of $\mathrm{TiO}_{6}$. When this distortion is treated as a static one, an orbital feels the following potential: $H_{J T}=-2\left|E_{J T}\right| Q_{i z}$, where $E_{J T}<0$ represents the total JT energy gain. In a radial gauge, we obtain the following phase Hamiltonian

$$
H_{\theta \varphi}^{J T}=\frac{9}{2} \rho_{0}\left|E_{J T}\right| \sum_{i}\left(\theta_{i}^{2}+\varphi_{i}^{2}\right) .
$$

Summing up all contributions, $H_{\theta \varphi}$ [Eq.(46)], $H_{\theta \varphi}^{\prime \prime}$, and $H_{\theta \varphi}^{J T}$, one obtains the following phase Hamiltonian:

$$
\begin{aligned}
H_{\theta \varphi}^{t o t} & =H_{\theta \varphi}+H_{\theta \varphi}^{\prime \prime}+H_{\theta \varphi}^{J T} \\
& =Z_{f} \sum_{\vec{q}}\left\{\frac{1}{2} \tilde{a}_{\theta}\left|\theta_{\vec{q}}\right|^{2}+\frac{1}{2} \tilde{a}_{\varphi}\left|\varphi_{\vec{q}}\right|^{2}-\gamma_{2}^{f} \varphi_{\vec{q}} \theta_{-\vec{q}}\right\},
\end{aligned}
$$

where $Z_{f}=\left(1+\left(\frac{t^{\prime \prime}}{t}\right)^{2}+3\left|E_{J T}\right|\right)$ is an overall factor. Here $\tilde{a}_{\theta}=1-\left(\gamma_{1}^{f}+\gamma_{3}^{f}\right)$ and $\tilde{a}_{\varphi}=1-\left(\gamma_{1}^{f}-\gamma_{3}^{f}\right)$, with modified form factors $\gamma_{n}^{f}=(1-2 f) \gamma_{n}(n=1,2,3)$ where $f=\left\{\left(\frac{t^{\prime \prime}}{t}\right)^{2}+\frac{3}{2}\left|E_{J T}\right|\right\} / Z_{f}$. 
Using now Eqs. (65) and (52) for $H_{\theta \varphi}$ and $H_{\lambda r}$, respectively, we obtain from $L_{\text {orb }}$ [Eq. (45)] the following excitation spectrum:

$$
\begin{aligned}
\omega_{ \pm}(\vec{k})=\{1-(1-2 \varepsilon)(1 & -2 f)\left(\gamma_{1} \pm \kappa\right)^{2} \\
& \left.-2(\varepsilon-f)\left(\gamma_{1} \pm \kappa\right)\right\}^{1 / 2} .
\end{aligned}
$$

This is given in units of $W_{\text {orb }}$, which is defined as follows:

$$
W_{\text {orb }}=\sqrt{Z_{\varepsilon} Z_{f}} r_{1} J_{S E} .
$$

$W_{\text {orb }}$ represents the overall energy scale for orbital fluctuations in the problem. Excitation gaps at $(0,0,0)$ and $(\pi, \pi, \pi)$ are given by $2 \sqrt{f(1-\varepsilon)} W_{\text {orb }}$ and $2 \sqrt{\varepsilon(1-f)} W_{\text {orb }}$, respectively. Taking $t^{\prime \prime} / t=0.2$ and $\left|E_{J T}\right|=0.04\left(r_{1} J_{S E}\right)$ as a representative values, we obtain $f=0.086$. With $\varepsilon=0.18$ and $\sqrt{Z_{\varepsilon}}=1.82$ estimated above (Sec. III D), one obtains $W_{\text {orb }} \simeq 1.96 r_{1} J_{S E}$, and the lowest gap about $0.53 W_{\text {orb }}$ is then expected at $(0,0,0)$ point. Thus, we expect that the orbital excitations in the modified model for $\mathrm{YTiO}_{3}$ cover the energy window from $\sim r_{1} J_{S E}$ to $\sim 2 r_{1} J_{S E}$.

\section{EFFECTIVE SPIN HAMILTONIAN}

The spin wave spectrum in $\mathrm{YTiO}_{3}$ shows the "cubic symmetry" of the Heisenberg spin couplings: $J_{a} \simeq J_{b} \simeq$ $J_{c} .{ }^{10}$ The magnon gap was found to be very small, almost two orders of magnitude smaller that the magnon bandwidth $(\sim 20 \mathrm{meV})$. It has been noticed that such an apparent simplicity of spin excitations, showing high isotropy in both real and spin spaces, is remarkable and puts strong constraints on possible orbital orderings. Spin wave excitations are examined in this section. Being a test case for the above theory for orbitals in $\mathrm{YTiO}_{3}$, a comparison with experiment gives also an opportunity to estimate SE energy scale $J_{S E}$ in the problem. To derive an effective Hamiltonian describing magnon excitations, we assume that orbital-spin separation occurs at low energies. This is justified when the orbital gap induced by Ti-O-Ti bond distortions (see Sec. V) is larger than magnon energy. Dynamical coupling between the spin and orbital degrees of freedom via fluctuations of superexchange bonds and also via on-site spin-orbital interaction $H_{s o}$ is then considered as a high-energy process, leading to an effective spin Hamiltonian. The parameters of such a Hamiltonian are obtained by integrating out high-energy orbital fluctuations.

\section{A. Isotropic spin exchange}

We start with estimation of coupling constant $J$ in the isotropic spin exchange term, $J\left(\vec{S}_{i} \cdot \vec{S}_{j}\right)$. As a first step, let us consider mean-field approximation, in which the spin exchange is given by an expectation value of the orbital operator in Eq. (2). Neglecting a small term $\frac{r_{2}-r_{3}}{3 r_{1}}\left\langle B_{i j}^{(\gamma)}\right\rangle$ and noticing that $\left\langle A_{i j}^{(\gamma)}\right\rangle=\frac{2}{3} E_{0}$, one obtains

$$
J_{0}=\left\{-\frac{2}{3} \eta r_{2}-\frac{2}{3}\left(1-\eta r_{2}\right)\left|E_{0}\right|\right\} r_{1} J_{S E} .
$$

The first term $(\sim \eta)$ is driven by a conventional Hund's coupling, while the second one originates from orbital singlet correlations in the ground state. For $\eta=0.12$, these two (classical and quantum) contributions are of the same order and give together $J_{0} \simeq-0.214\left(r_{1} J_{S E}\right)$.

However, the actual value of $J$ measured experimentally from magnon spectra could in fact be strongly reduced from $J_{0}$ in Eq. (68) due to a fluctuation effects. Indeed, $\mathrm{AF}$ and $\mathrm{F}$ states are strongly competing in $t_{2 g}$ systems, and large-scale orbital fluctuations are expected to bring about AF spin exchange contribution. We, therefore, have to consider effects of the dynamical spin-orbital interaction:

$$
H_{i n t}=\sum_{\langle i j\rangle} \delta\left(\vec{S}_{i} \cdot \vec{S}_{j}\right) \delta \hat{J}_{i j} .
$$

In a ferromagnetic state $\delta\left(\vec{S}_{i} \cdot \vec{S}_{j}\right) \simeq-\frac{1}{2}\left(s_{i}^{\dagger}-s_{j}^{\dagger}\right)\left(s_{i}-s_{j}\right)$, with $s_{i}^{\dagger}$ being a magnon creation operator. Neglecting small $\frac{r_{1}-r_{2}}{2 r_{1}}$ and $\frac{r_{2}-r_{3}}{3 r_{1}}$ terms in Eq.(2), exchange integral fluctuations are given by $\delta \hat{J}_{i j} \simeq \delta A_{i j}^{(\gamma)}$ (in units of $\left.r_{1} J_{S E}\right)$. As the coupling constant in Eq. (69) is not small, and because of spins and orbitals may form bound states $^{4}$ in an excited AF states, we will discuss here only a qualitative picture. We introduce a correlation function $D_{i j}^{(\gamma)}(\tau)=\left\langle T_{\tau} \delta \hat{J}_{i j}(0) \delta \hat{J}_{i j}(\tau)\right\rangle$ describing fluctuations of the spin exchange integral. We assume that its spectral function $\rho(\omega)=\frac{1}{\pi} D_{i j}^{\prime \prime}(\omega+i \delta)$ is distributed over the characteristic energies larger than low-energy coherent magnons observed in the experiment (an adiabatic approximation which is valid as far as one is concerned with low-energy spin excitations). Within this approximation and neglecting vertex corrections we may evaluate the magnon scattering process on a given bond as described in Fig. 9. The result implies a renormalization of the coupling constant in low energy spin Hamiltonian by $\delta J_{\text {eff }}=\frac{1}{2} D_{i j}(0)=\int_{0}^{\infty} \rho(\omega) \frac{d \omega}{\omega}$, which is of AF sign as expected. It is the renormalized exchange coupling $J=J_{0}+\delta J_{\text {eff }}$ that determines magnon spectra. We can estimate $D_{i j}(\omega)$ by keeping in $\delta \hat{J}_{i j} \simeq \delta A_{i j}$ [Eq. (23)] the orbiton pair excitation terms only:

$$
\delta \hat{J}_{i j}^{(c)}(\text { pair })=\frac{1}{3}\left(\tilde{a}_{i}^{\dagger} \tilde{a}_{j}^{\dagger}+\tilde{b}_{i}^{\dagger} \tilde{b}_{j}^{\dagger}-\tilde{a}_{i}^{\dagger} \tilde{b}_{j}^{\dagger}-\tilde{b}_{i}^{\dagger} \tilde{a}_{j}^{\dagger}+\text { H.c. }\right) .
$$

We expect that orbiton pair fluctuations are rather incoherent and local, and we parametrize their spectral function by a characteristic energy $\Omega_{\text {pair }}$, obtaining

$$
D_{i j}(i \nu)=\frac{4}{9} \frac{2 \Omega_{\text {pair }}}{\Omega_{\text {pair }}^{2}+\nu^{2}},
$$




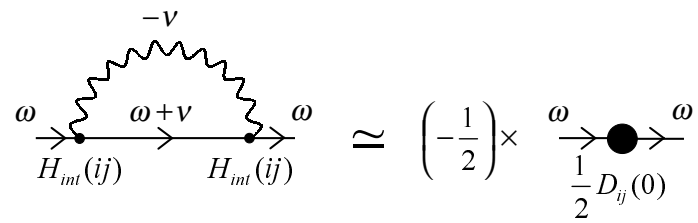

FIG. 9. Scattering of low energy magnons on local fluctuations of the spin exchange integral, $\delta \hat{J}_{i j}$. Its Green's function (wavy line) is taken at zero frequency (adiabatic approximation, see text). This results in an additional effective spin exchange constant (denoted by a filled circle on right hand side), $\delta J_{e f f}=\frac{1}{2} D_{i j}(0)$ for low energy magnons.

thus $\delta J_{\text {eff }}=\frac{4}{9} \frac{1}{\Omega_{\text {pair }}}$. We notice that Eq. (71) means also that $\left\langle\left(\delta J_{i j}\right)^{2}\right\rangle=\frac{4}{9}$, which can be simply understood as follows: on a given exchange bond, e. g., along the $c$ axis, one may have in general nine orbital configurations. ¿From Eq. (11) one observes that these are the orbital singlet giving ferromagnetic spin exchange $J=-1$, orbital triplet states with $J=+1$, and five states with $n_{i}^{(\gamma)} n_{j}^{(\gamma)}=0$ giving zero $J$. As orbital order is weak, and because of the formation of orbital singlets/triplets at given bond necessarily frustrates other neighboring bonds, all these configurations will be present giving $\left\langle\left(\delta J_{i j}\right)^{2}\right\rangle \sim \frac{4}{9}$. It is also natural to expect that $\Omega_{\text {pair }} \sim$ $W_{\text {orb }}$, with $W_{\text {orb }} \sim 2\left(r_{1} J_{S E}\right)$ as estimated in the preceding section. This gives an estimation $\delta J_{\text {eff }} \simeq \frac{2}{9}$ (in units of $\left.r_{1} J_{S E}\right)$, resulting finally in effective exchange coupling for low energy spin excitations as follows:

$$
J \simeq\left\{-\frac{2}{3} \eta r_{2}-\frac{2}{3}\left(1-\eta r_{2}\right)\left|E_{0}\right|+\frac{2}{9}\right\} r_{1} J_{S E} .
$$

These qualitative estimations are substantiated in Appendix $\mathrm{D}$, in which we calculate magnon energy renormalization within a linear orbital wave theory.

It is observed from Eq. (72) that $J$ is actually positive (antiferromagnetic) for realistic values of $\eta \sim 0.12-0.13$, in agreement with the conclusion obtained above from energy considerations: The ground state of the model is not ferromagnetic in an ideal cubic lattice. At the presence of Ti-O-Ti bond angle distortion, $J$ is however modified as follows: Eq. (72) obtains a prefactor $\cos ^{2} \theta$, and in addition a term $-J^{\prime} \simeq-\frac{2}{3} \eta \sin ^{2} \theta$ [Eq. (58)] has to be accounted for. As a result, a classical Hund's rule part of $J$ remains unchanged, and the net result

$J=\left[-\frac{2}{3} \eta r_{2}+\cos ^{2} \theta\left\{\frac{2}{9}-\frac{2}{3}\left(1-\eta r_{2}\right)\left|E_{0}\right|\right\}\right] r_{1} J_{S E}^{(0)}$

gives a small ferromagnetic coupling $J \simeq-0.03 r_{1} J_{S E}^{(0)}$ for $\mathrm{YTiO}_{3}$ with $\theta \approx 142$ deg. Comparing this result (at $\eta=0.12$ ) with experimental one $J_{\text {exp. }}=-2.75 \mathrm{meV},{ }^{10}$ we obtain the overall energy scale $r_{1} J_{S E}^{(0)} \simeq 92 \mathrm{meV}$ and $J_{S E}^{(0)}=4 t_{0}^{2} / U \simeq 59 \mathrm{meV}$. Bond distortion effect reduces the energy scale to $r_{1} J_{S E} \sim 78 \mathrm{meV}$ and $\sim 57 \mathrm{meV}$ in La and $\mathrm{Y}$ based titanates, respectively. Based on the above considerations, we consider $J_{S E}^{(0)} \sim 60 \mathrm{meV}$ and $r_{1} J_{S E} \sim$ $60 \mathrm{meV}$ as representative energy scales for $\mathrm{YTiO}_{3}$.

A main message of the above considerations is that the spin-exchange constant as seen by a coherent lowenergy magnon excitations in $\mathrm{YTiO}_{3}$ represents in fact only a small fraction of the real strength of dynamical spin couplings. Because the sign of $t_{2 g}$-spin exchange is not unique, and because the orbital order is weak, large fluctuations of the spin couplings are present in titanates.

\section{B. Anisotropic SE interaction}

Next, we consider effects of a relativistic spin-orbit coupling

$$
H_{s o}=\lambda \sum_{i}\left(\vec{S}_{i} \cdot \vec{l}_{i}\right)
$$

This interaction introduces anisotropy in the effective spin Hamiltonian, which obtains (besides the rotationally invariant Heisenberg part) an additional, so-called antisymmetric Dzyaloshinskii-Moriya (DM) and symmetric anisotropy interactions. ${ }^{22}$ The anisotropic interactions select orientation of the magnetization in the crystal, and lead also to magnon gap(s). The structure of anisotropic terms is essentially determined by orbital state via expectation values and dynamics of the angular momentum operator in Eq.(74). Thus, we would like to obtain spinorbit coupling induced corrections to the spin Hamiltonian, and discuss their consequences on magnon spectra, thereby testing the proposed orbital state.

As usual, anisotropic interactions are obtained by perturbation theory involving both isotropic $H_{S E}$ and $H_{s o}$. We mostly discuss the quadrupole ordered orbital state with condensed $\tilde{c}$ orbitals (see Sec. III B). In derivation of the anisotropy Hamiltonian, we need to keep in the superexchange operators $\hat{J}_{i j}^{(\gamma)}$ and $\hat{K}_{i j}^{(\gamma)}$ in Eq.(1) such terms that (i) operate in the $\tilde{a} \tilde{b}$ excited states and/or (ii) connect a ground state with excited states of orbitals. For instance,

$$
\begin{gathered}
\hat{J}_{i j}^{(c)} \Rightarrow J_{S E} \frac{r_{1}+r_{2}}{6}[ \\
{\left[n_{i \tilde{a}}+n_{j \tilde{a}}+\tilde{a}_{i}^{\dagger} \tilde{a}_{j}^{\dagger}+\tilde{a}_{i} \tilde{a}_{j}-\tilde{a}_{i}^{\dagger} \tilde{b}_{j}^{\dagger}-\tilde{a}_{i} \tilde{b}_{j}\right.} \\
+(a \leftrightarrow b)] .
\end{gathered}
$$

[Here, the terms proportional to small numbers $\left(r_{1}-r_{2}\right)$ and $\left(r_{2}-r_{3}\right)$ are neglected].

We consider nearest-neighboring sites $i$ and $j$. The local excitation energy to create an orbiton $\tilde{a}$ or $\tilde{b}$ is denoted as $\Delta_{l o c}$. It is reasonable to associate $\Delta_{l o c}$ with the "center of gravity" of the orbiton band that covers the energy window from $\sim r_{1} J_{S E}$ to $\sim 2 r_{1} J_{S E}$ as obtained in Sec. V. Thus, we will consider $\Delta_{l o c} \sim 1.5 r_{1} J_{S E}$ in our estimations, when we compare later on the results with experiment.

To obtain spin anisotropy interactions, it is convenient to work again in a rotated basis, applying transformation 
(19) also for the spins. The scalar product of NN spins in $H_{S E}$ is then expressed as $\vec{S}_{i} \cdot \vec{S}_{j}=\overrightarrow{\widetilde{S}}_{i} \widetilde{T}^{(\gamma)} \overrightarrow{\widetilde{S}}_{j}$ where

$$
\widetilde{T}^{(a)}=\frac{1}{3}\left(\begin{array}{rrr}
-1 & 2 & -2 \\
2 & -1 & -2 \\
-2 & -2 & -1
\end{array}\right)
$$

and

$$
\widetilde{T}^{(b, c)}=\frac{1}{3}\left(\begin{array}{rrr}
-(1 \mp 2 s) & -1 & 1 \pm 2 s \\
-1 & -(1 \pm 2 s) & 1 \mp 2 s \\
1 \pm 2 s & 1 \mp 2 s & -1
\end{array}\right)
$$

for the state I (a). [For the state I (b), matrices $\widetilde{T}$ for bonds $a$ and $b$ are equal and given by Eq. (76)].

Third-order perturbation with respect to $H_{s o}$ and $H_{S E}$ gives a symmetric part of the spin anisotropy Hamiltonian $H_{a n i}$ in a local coordinate:

$$
H_{a n i}^{(\gamma)}=-\frac{1}{2} A \overrightarrow{\widetilde{S}}_{i} \widetilde{M}^{(\gamma)} \overrightarrow{\widetilde{S}}_{j}
$$

with

$$
\widetilde{M}^{(c)}=\frac{1}{2}\left(\begin{array}{rrr}
2 & 1 & c-3 s \\
1 & 2 & c+3 s \\
c-3 s & c+3 s & -1
\end{array}\right) .
$$

Anisotropy constant $A$ in Eq. (78) is given by the following expression:

$$
A=\frac{4}{9} J_{S E} \frac{r_{1}+r_{2}}{2}\left(\frac{\lambda}{\Delta_{l o c}}\right)^{2} .
$$

Transformation of the spin operator from a local to global coordinates is expressed as $\vec{S}_{i}=\hat{R}_{i} \hat{R} \overrightarrow{\widetilde{S}}_{i}$. The matrices $\hat{R}_{i}$ $(i=1,2,3$ and 4$)$ transforming spin coordinates at four sublattices are as follows:

$$
\begin{gathered}
\hat{R}_{1}=\left(\begin{array}{lll}
1 & 0 & 0 \\
0 & 1 & 0 \\
0 & 0 & 1
\end{array}\right), \hat{R}_{2}=\left(\begin{array}{ccc}
-1 & 0 & 0 \\
0 & -1 & 0 \\
0 & 0 & 1
\end{array}\right), \\
\hat{R}_{3}=\left(\begin{array}{ccc}
-1 & 0 & 0 \\
0 & 1 & 0 \\
0 & 0 & -1
\end{array}\right), \hat{R}_{4}=\left(\begin{array}{ccc}
1 & 0 & 0 \\
0 & -1 & 0 \\
0 & 0 & -1
\end{array}\right) .
\end{gathered}
$$

Using this transformation, one obtains a symmetric anisotropy Hamiltonian defined in the global coordinate:

$$
H_{a n i}^{(i j)}=-A \vec{S}_{i} \hat{M}_{i j} \vec{S}_{j},
$$

where $\hat{M}_{i j}$ depends on the NN bond. For 1-3 (2-4) bonds along the $c$ axis, $\hat{M}_{13(24)}$ is given by

$$
\hat{M}_{13(24)}=\frac{1}{4}\left(\begin{array}{rrr}
3 / 2 & 0 & \pm 3 \\
0 & 7 / 2 & 0 \\
\pm 3 & 0 & -3 / 2
\end{array}\right) .
$$

The interaction matrices for NN spins on $a, b$ bonds have similar structure:

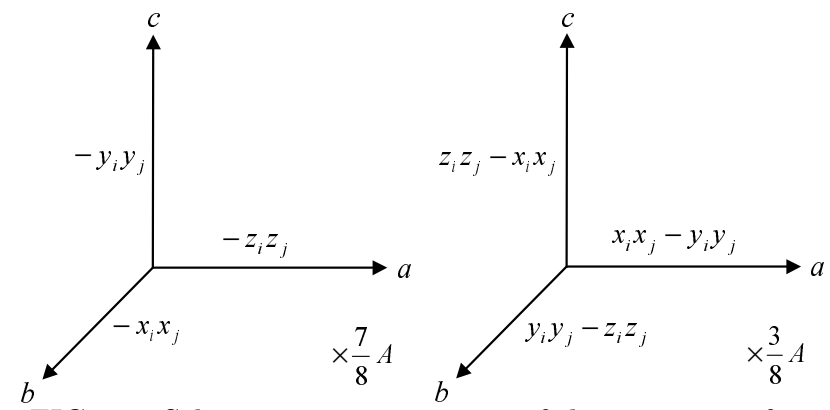

FIG. 10. Schematic representation of the structure of symmetric spin anisotropy interactions of $e_{g}$-symmetry. Interactions along different bonds are denoted by $\alpha_{i} \alpha_{j}$, which should read as $S_{i \alpha} S_{j \alpha}$ times an overall interaction constant given below each figure. For instance, $a$-bond interactions are $-\frac{7}{8} A S_{i z} S_{j z}$ and $\frac{3}{8} A\left(S_{i x} S_{j x}-S_{i y} S_{j y}\right)$ (note $e_{g}\left(3 z^{2}-r^{2}\right)$ - and $e_{g}\left(x^{2}-y^{2}\right)$-type symmetry), where the constant $A$ is defined by Eq.(80). Overall cubic symmetry of the interactions is evident for both contributions.

$$
\begin{aligned}
& \hat{M}_{12(34)}=\frac{1}{4}\left(\begin{array}{rrr}
-3 / 2 & \pm 3 & 0 \\
\pm 3 & 3 / 2 & 0 \\
0 & 0 & 7 / 2
\end{array}\right) \\
& \hat{M}_{14(23)}=\frac{1}{4}\left(\begin{array}{rrr}
7 / 2 & 0 & 0 \\
0 & -3 / 2 & \pm 3 \\
0 & \pm 3 & 3 / 2
\end{array}\right) .
\end{aligned}
$$

Symmetric anisotropy interactions can be classified according to cubic invariants: there are terms of $e_{g}$ and $t_{2 g}$ symmetries, generated by diagonal and nondiagonal elements of the matrices $\hat{M}_{i j}$, correspondingly. For convenience, we show the interactions for the state I (a) in Fig. 10 and Fig. 11 (a), in which bond dependence, direction of spins and the scale of individual anisotropy terms are shown. The $e_{g}$ symmetry anisotropy in Fig. 10 (a) has been discussed in Ref. 13 under the name of "cubic" anisotropy in the context of magnon gap in $\mathrm{LaTiO}_{3}$. A remarkable feature of this interaction is its intrinsic frustrations: namely, treated classically, it acquires a rotational symmetry in spin sector, resulting in an infinite degeneracy of classical states. An accidental pseudoGoldstone mode, which appears in classical limit, can acquire finite gap by quantum fluctuations only. In the present orbital ordered states, the symmetry is lowered compared with the orbital liquid state in $\mathrm{LaTiO}_{3}$. Thus, additional terms are generated as shown in Figs. 10 (b) and 11 (a). It is noticed that these terms have a similar frustrated nature: summed over all the bonds, they cancel each other exactly. Thus, only a small gap is expected from these interactions.

Physically, the structure of anisotropy interactions is determined by local correlations of the angular momentum, and can therefore be traced back to noncollinear arrangements of these correlations shown in Fig. 2 (a). For instance, the leading, "cubic" term [see Fig. 10 (a)] reflects that $l_{y}$ components are correlated ferromagnetically along the $c$ axis, while $l_{z}\left(l_{x}\right)$ components are parallel along $a(b)$ axes. 

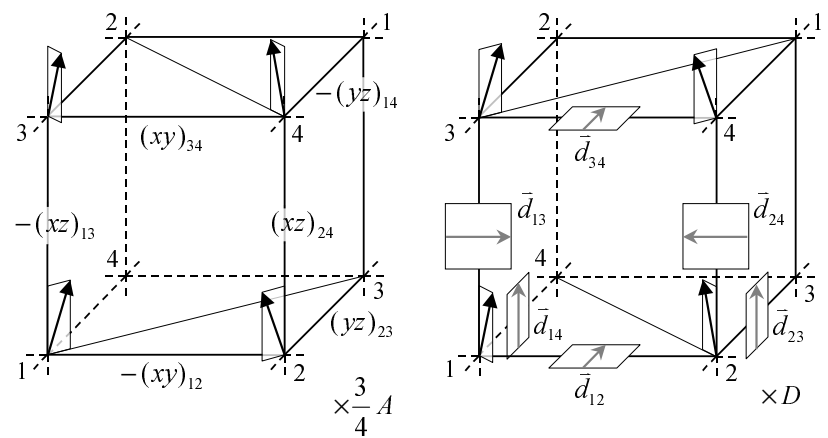

FIG. 11. Left: Schematic representation of the structure of symmetric spin anisotropy interactions of $t_{2 g}$-symmetry. The notation $(\alpha \beta)_{i j}$ stands for $S_{i \alpha} S_{j \beta}+S_{i \beta} S_{j \alpha}$ multiplied by interaction constant $3 A / 4$. Black arrows represent the direction of spins favorable for this interaction when the direction of the uniform moment is taken along [001]. Right: Antisymmetric DM spin anisotropy interactions [Eq.(88)]. Gray arrow denoted by $\vec{d}_{i j}$ shows the orientation of DM vectors on different bonds. A preferred spin pattern for this interaction is shown by black arrows.

In the state I (b), the leading anisotropy interaction in the $a b$-plane is given by $-S_{i}^{z} S_{j}^{z}$ and that along $c$-direction is obtained to be $-S_{i}^{y} S_{j}^{y}$ [as can easily be seen also from Fig. 2 (b)]. The number of bonds with anisotropic interaction $-S_{i}^{z} S_{j}^{z}$ is two times larger than that with $-S_{i}^{y} S_{j}^{y}$. This breaks the rotational symmetry in a spin sector even in the classical limit, and generates a large magnon gap $\Delta_{\text {mag }}=\frac{7}{4} \sqrt{2} S A$.

Antisymmetric DM interaction appears in a secondorder perturbation theory as a combined effect of $H_{S E}$ and $H_{\text {so }}$. The remarkable feature of the orbital state $\mathrm{I}$ is that leading terms in SE interactions, which are proportional to $\left(r_{1}+r_{2}\right)$ in Eq.(2), do not contribute to the DM interaction. That is because of the classical expectation value of $A_{i j}$ in Eqs. (4) and (8) vanishes in our orbital states. Rather much smaller Hund's coupling terms proportional to the small number $\left(r_{1}-r_{2}\right)$ only give rise to DM interaction. This feature contrasts with that in the orbital state reported in Refs. 15, 23-26, in which a large DM interaction is present (see Appendix E).

After somewhat tedious but straightforward calculations one obtains the following interaction between NN sites:

$$
H_{D M}^{(\gamma)}=D \overrightarrow{\widetilde{S}}_{i} \widetilde{N}^{(\gamma)} \overrightarrow{\widetilde{S}}_{j}
$$

Here, the matrix $\widetilde{N}^{(c)}$ reads as

$$
\tilde{N}^{(c)}=\frac{1}{3}\left(\begin{array}{rrr}
2 c-2 s & 1 & c+s \\
1 & 2 c+2 s & c-s \\
c+s & c-s & -2
\end{array}\right)
$$

and the interaction constant $D$ is obtained as follows:

$$
D=J_{S E} \frac{r_{1}-r_{2}}{6} \frac{\lambda}{\Delta_{l o c}} .
$$

Transforming the local spin axes to the global ones, one arrives at the following DM interaction:

$$
H_{D M}^{(i j)}=D \vec{d}_{i j} \cdot\left(\vec{S}_{i} \times \vec{S}_{j}\right)
$$

with $\vec{d}_{i j}=\hat{\alpha}_{i}^{\prime}$. Here, $\hat{\alpha}_{i}$ is the unit vector parallel to one of local axes $\left(x_{i}, y_{i}\right.$ and $\left.z_{i}\right)$ which is perpendicular to the $i-j$ bond direction and antiparallel to its counterpart at site $j$ [see Fig. 2 (a)]. For example, $\vec{d}_{13}=(1,0,0)$, $\vec{d}_{12}=(0,1,0), \vec{d}_{14}=(0,0,1)$, etc. For convenience, we show the DM interaction for state I (a) in Fig. 11 (b).

\section{Spin waves: comparison with experiment}

Now, we discuss the anisotropic spin interactions in the context of the experimental observations of magnon dispersion in $\mathrm{YTiO}_{3} \cdot{ }^{10}$

Cubic symmetry of the spin wave dispersion: This puzzling observation is naturally explained by the present theory, as ferromagnetic couplings in all the states I and II are perfectly isotropic, $J^{(a)}=J^{(b)}=J^{(c)}$. The reason is high symmetry of the orbital ordering patterns, as can be visualized from Fig. 4. It is stressed that this result is robust, "no fine-tuning" property of the model. (The isotropy is expected to be relaxed somewhat by lattice distortions. However, effects of two types of distortions, that is, Ti-O-Ti bond angle distortion and elongation of $\mathrm{TiO}_{6}$ octahedron, on the anisotropy of spin couplings are opposite and almost cancel each other. ${ }^{27}$ )

Isotropy in spin space, magnon gap:

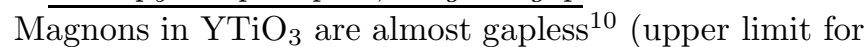
the gap is $0.3 \mathrm{meV}$ ); this is a serious test for possible orbital orderings. We show now that high symmetry of orbital orderings in the present theory resolves this problem as well. The crucial point is the frustrating nature of the anisotropic spin interactions obtained above: Even though anisotropic couplings on an individual bond are substantial, there is cancellation of classical contributions stemming from different bonds, and one obtains only small gaps of quantum origin. We illustrate this by considering first the leading "cubic" term.

Quantum magnon gap by "cubic" term: Consider the effective spin Hamiltonian in the state I (a) given as follows:

$$
\begin{aligned}
H_{s} & =H_{i s o}+H_{a n i} \\
& =-\sum_{\langle i j\rangle}\left[J \vec{S}_{i} \cdot \vec{S}_{j}+\tilde{A} S_{i}^{(\gamma)} S_{j}^{(\gamma)}\right] .
\end{aligned}
$$

Here, $H_{\text {iso }}$ represents the isotropic SE Hamiltonian. Coefficient of the "cubic" term is $\tilde{A}=7 / 8 A$ [see Fig. 10 (a)], and axes in the spin space are changed as $S^{z} \rightarrow S^{x}$, $S^{x} \rightarrow S^{y}$ and $S^{y} \rightarrow S^{z}$ such that $\gamma$ corresponds to the direction of the $i-j$ pair. Thus, we have $-S_{i}^{z} S_{j}^{z}$ for $c$ bonds, $-S_{i}^{x} S_{j}^{x}$ for $a$-bonds and $-S_{i}^{y} S_{j}^{y}$ for $b$-bonds. 
Because the effective spin Hamiltonian $H_{s}$ has a discrete (cubic) symmetry, magnon excitation is expected to have a gap. However, due to the rotational symmetry of Eq. (89) in the limit of classical spins, linear spin-wave theory cannot provide finite gap. This problem is resolved by the order-from-disorder mechanism, ${ }^{19}$ which selects a particular classical state which provides the largest zero-point energy when fluctuations are included. This opens also a magnon gap. ${ }^{13,28}$ Thus, we calculate spin-wave contribution to the ground-state energy as a function of the angle $\theta$ between the $c$ axis and the uniform moment. First, we rotate the spin quantization axes around the $b$ axis:

$$
\begin{aligned}
& S_{i}^{x}=c_{\theta} \widetilde{S}_{i}^{x}+s_{\theta} \widetilde{S}_{i}^{z}, \\
& S_{i}^{z}=-s_{\theta} \widetilde{S}_{i}^{x}+c_{\theta} \widetilde{S}_{i}^{z},
\end{aligned}
$$

where $c_{\theta}=\cos \theta$ and $s_{\theta}=\sin \theta$. Second, by using Holstein-Primakoff approximation, we obtain the magnon dispersion which shows explicit $\theta$ dependence as

$$
\omega_{\vec{k}}(\theta)=z S J \sqrt{X_{\vec{k}}\left[Y_{\vec{k}}-a\left(c_{z}-c_{x}\right) s_{\theta}^{2}\right]},
$$

where $X_{\vec{k}}=\left(1-\gamma_{1}\right)+a\left(1-c_{y}\right), Y_{\vec{k}}=\left(1-\gamma_{1}\right)+a\left(1-c_{x}\right)$, and $a=\tilde{A} / 3 J$ represents the ratio between anisotropic and isotropic interactions. Finally, by calculating the zero point magnon energy, we obtain the ground-state energy $E_{0}$ per site. In the limit of $\theta \ll 1$,

$$
\begin{aligned}
E_{0}(\theta) & =-\frac{1}{2} z J S(S+1)(1+a)+\frac{1}{2} \sum_{\vec{k}} \omega_{\vec{k}}(\theta) \\
& =- \text { const }+K_{e f f} S^{2} \theta^{2} .
\end{aligned}
$$

Here, $K_{\text {eff }}$ represents an effective spring constant. In the case of $a \ll 1$ which we are interested in, the spring constant is given by $K_{\text {eff }}=\tilde{A}^{2} R / z S J$ with

$$
R=3 \sum_{\vec{k}} \frac{\gamma_{2}^{2}}{1-\gamma_{1}} \approx 0.28 .
$$

The potential $E_{0}(\theta)$ in Eq.(92) can be associated with an effective uniaxial spin anisotropy Hamiltonian

$$
H_{e f f}^{a n i}=-K_{\text {eff }} \sum_{\langle i j\rangle_{c}} S_{i}^{z} S_{j}^{z},
$$

generated in the symmetry-broken phase (with spins oriented along [001]). Therefore, one finds a magnon gap $\Delta_{m a g}=2 S K_{\text {eff }}=2 \tilde{A}^{2} R / z J$. Note that $\Delta_{m a g}$ is independent of $S$ and is proportional to $\tilde{A}^{2}$. We confirmed that the same magnitude of the magnon gap is derived from $H_{s}$ using single mode approximation ${ }^{29}$ (see Appendix $\mathrm{C}$ ). Thus, "cubic" anisotropy gives the magnon gap $\Delta_{\text {mag }}=2\left(\frac{7}{8} A\right)^{2} R / z J$.

Now, we estimate the coupling constant $A$. ¿From Eq. (80) with $r_{1} J_{S E} \sim 60 \mathrm{meV}$ and $\eta \sim 0.12$, one finds $A \simeq 23\left(\lambda / \Delta_{l o c}\right)^{2}$. As discussed in the precedings section, we consider $\Delta_{l o c} \sim 1.5 r_{1} J_{S E} \sim 90 \mathrm{meV}$. Using the atomic value $\lambda_{a t}=19 \mathrm{meV},{ }^{30}$ one obtains then $A \simeq 1 \mathrm{meV}$ consistent with the experimental value of $A$ obtained in Ref. 10 from magnon spectra. With this value of $A$, the magnon gap from the "cubic" term is only $\approx 0.03 \mathrm{meV}$ in the state I (a).

On the other hand, a large classical magnon gap $\Delta_{\text {mag }}=\frac{7}{4} \sqrt{2} S A \approx 1.2 \mathrm{meV}$ is obtained in the state I (b), as we already discussed in the preceding section. This allows us to exclude the orbital ordering structure I (b) from possible candidates for $\mathrm{YTiO}_{3}$, although we do not know precisely which kind of lattice distortions favor state I (a) over configuration I (b).

Contributions from the other terms: Anisotropy of $e_{g}\left(x^{2}-y^{2}\right)$ symmetry [see Fig. 10 (b)] can be analyzed similarly; we find that it also supports an easy magnetization axis along one of the cubic axes, say [001]. Its contribution to the magnon gap, $\Delta_{\text {mag }}=6\left(\frac{3}{8} A\right)^{2} R / z J \simeq$ $0.014 \mathrm{meV}$ is smaller than that of the "cubic" term, as expected.

Once the [001] direction is chosen as the direction of uniform moment, spin anisotropy terms of $t_{2 g}$ symmetry and DM interactions give rise to spin cantings as shown in Figs. $11(\mathrm{a}, \mathrm{b})$. The canting angle $\theta$ is given by $\theta \approx(3 / 2 \sqrt{2})(A / 4 J) \simeq 0.1 \mathrm{rad}$ for $t_{2 g}$ symmetric anisotropy interaction, and $\theta \approx \sqrt{2}(D / 4 J) \simeq 0.07 \mathrm{rad}$ for the antisymmetric DM interaction. [DM interaction constant $D \approx 0.57 \mathrm{meV}$ is estimated from Eq.(87)]. These values are within the experimental canting angles $\simeq 0.17$ rad. ${ }^{10}$ Finally, contributions of $t_{2 g}$ symmetric anisotropy and DM interactions to the magnon gap $\Delta_{\text {mag }}$ are estimated as $\approx 4 J S \theta^{2}\left(t_{2 g}\right) \sim 0.05 \mathrm{meV}$ and $\approx 4 J S \theta^{2}(D M) \sim 0.03 \mathrm{meV}$, correspondingly; these numbers are rather small again. It should be noticed, that a more quantitative analysis of the problem, in particular the precise structure of the spin canting pattern requires consideration of all the anisotropy terms on equal footing, which will be presented elsewhere.

To summarize this section, we have obtained spin anisotropy interactions induced by the spin-orbit coupling, and considered their effects on spin-wave spectra. Because of high symmetry of the orbital ordering, particularly in the quadrupole ordered state I (a), magnon dispersion is found to have cubic symmetry, magnon gap is small, and there are small cantings of spins away from the $c$ axis. All these observations are consistent with experiment.

\section{ORBITAL CONTRIBUTION TO THE RESONANT X-RAY SCATTERING}

We turn to the discussion of further experiments which may help to verify the proposed orbital state in $\mathrm{YTiO}_{3}$. First, we consider the resonant x-ray scattering, which has proven to be a useful method in the study of or- 
bital order symmetry. ${ }^{31,32}$ The following section will be devoted to possible ways of detecting orbital excitations.

We focus on the orbital state I (a) [which is the most plausible candidate as discussed in previous sections]. While the exchange bonds in this state are the same (important for the isotropy of spin waves), a local symmetry is lower than a cubic one [see Fig.(4)]. Thus, orbital order may induce spatial modulations of the level structure of an excited photoelectron in $p$-states via the so-called Coulomb mechanism. ${ }^{32}$ This may lead to additional weak reflections at orbital ordering vectors. Predictions of our theory for such an experiment are as follows. Orbital order shown in Fig. 4(I) is identified as a three-component quadrupole ordering of $t_{2 g}$-symmetry, $T_{\alpha}(\vec{q})$ with $\alpha=x, y, z$. Each component has its own propagation vector:

$$
\begin{aligned}
& T_{x}=\left\langle l_{y} l_{z}+l_{z} l_{y}\right\rangle_{\vec{R}}=\frac{2}{3} Q e^{i \vec{q}_{1} \cdot \vec{R}}, \\
& T_{y}=\left\langle l_{x} l_{z}+l_{z} l_{x}\right\rangle_{\vec{R}}=\frac{2}{3} Q e^{i \vec{q}_{2} \cdot \vec{R}}, \\
& T_{z}=\left\langle l_{x} l_{y}+l_{y} l_{z}\right\rangle_{\vec{R}}=\frac{2}{3} Q e^{i \vec{q}_{3} \cdot \vec{R}},
\end{aligned}
$$

where $\vec{q}_{1}=(\pi, 0, \pi), \vec{q}_{2}=(\pi, \pi, 0)$, and $\vec{q}_{3}=(0, \pi, \pi)$. As order parameter $Q$ is strongly suppressed by quantum fluctuations $(Q \simeq 0.19$, see Sec. III B), we obtain that each component has only a small amplitude, giving $\left|T_{\alpha}\right|^{2} \sim 0.016$. This implies that the corresponding anomalous Bragg intensity is at least 60 times weaker compared with the classical orbital orderings. It might therefore be very difficult to single out this contribution. However, new azimuthal $(\varphi)$ and scattering $\left(\theta_{s}\right)$ angular dependencies of an additional intensity, which should show up below $T_{\text {orb }}$, may help to identify order symmetry. For $(\pi, \pi, 0)[(100)$ in orthorhombic notations] scattering these dependences are obtained as follows

$$
I_{\sigma \sigma^{\prime}}(\pi, \pi, 0) \propto \sin ^{2} 2 \varphi
$$

for $\sigma-\sigma^{\prime}$ polarization (see for notations Ref. 31), and

$$
I_{\sigma \pi^{\prime}}(\pi, \pi, 0) \propto\left(\cos 2 \varphi \sin \theta_{s}+\sin \varphi \cos \theta_{s}\right)^{2}
$$

for $\sigma-\pi^{\prime}$ polarization. (Azimuthal angle $\varphi=0$ corresponds to the configuration in which the diffraction plane is parallel to the $c$ axis.) Scattering intensities at $\vec{q}_{1}$ and $\vec{q}_{3}$ (which are contributed by $T_{x}$ and $T_{z}$ components, respectively) can be obtained from symmetry considerations.

It is still a controversial issue whether the resonant $\mathrm{x}$-ray scattering, observed in $\mathrm{YTiO}_{3},{ }^{26}$ is related to orbital order or lattice distortions (see Refs. 33, 26, 17). Either way, we expect that the orbital order contribution, if present, must be temperature dependent reflecting orbital order/disorder transition, as in the case of manganites with strong orbital order. Therefore, a careful analysis of the $T$-dependence of reflections at orbital ordering vectors is desirable.
Above discussion brings us to the problem of the orbital ordering temperature in $\mathrm{YTiO}_{3}$. Thus far, there are no reports on the orbital ordering temperature in $\mathrm{YTiO}_{3}$ (weak structural change at spin ordering temperature ${ }^{26}$ is only an indirect indication). In our SE-model picture, we expect that this transition should occur at low temperatures only, and we suspect in fact that orbitals in $\mathrm{YTiO}_{3}$ do order at ferromagnetic spin transition $T_{C}$. This is because of strong spin/orbital coupling, and also because of the frustrated nature of orbital-only model itself, which, as we have shown, may develop long-range order on a cubic lattice at zero temperature only. Lattice distortions that open a finite orbital gap allow finite-temperature transition, but this cannot occur much above the ferromagnetic transition, because of strong disorder introduced by spin fluctuations in the paramagnetic phase. In other words, orbital order and isotropic spin ferromagnetism are intimately connected, supporting each other. Physically, this implies that short-range ferromagnetic correlations are of vital importance for orbital ordering, and vice versa.

A quantitative description of the finite-temperature behavior of a realistic spin-orbital model is complicated. We may give only very rough estimation for the orbital ordering temperature based on the mean-field picture. As we are going to ignore fluctuations completely, this estimation should be regarded as an upper limit, which we would like to know. To this end, we consider a spin paramagnetic phase and set $\left\langle\vec{S}_{i} \cdot \vec{S}_{j}\right\rangle=0$ in Eq. (1), neglect in the orbital interactions $A_{i j}^{(\gamma)}$ in Eq. (23) all the terms except those which contain an emerging quadrupole order parameter. This leads to

$$
\begin{array}{r}
H_{o r b}=-\frac{1}{3 z} \sum_{\langle i j\rangle} \hat{Q}_{i z} \hat{Q}_{j z} \\
\Rightarrow-\frac{1}{3}\langle\hat{Q}\rangle \hat{Q}_{i z} .
\end{array}
$$

From Eq. (98) we obtain $T_{\text {orb }}=\frac{1}{6}$ (in units of $r_{1} J_{S E}$ ), which, including the Ti-O-Ti bond angle $(\theta \simeq 142 \mathrm{deg})$ correction for $\mathrm{YTiO}_{3}$, reads as

$$
T_{\text {orb }}=\frac{1}{6} \cos ^{2} \theta\left(r_{1} J_{S E}^{(0)}\right) \simeq 0.1\left(r_{1} J_{S E}^{(0)}\right) .
$$

On the other hand, spin ordering (mean-field) temperature is $T_{C}=\frac{3}{2}|J|$, with $J \simeq-0.03\left(r_{1} J_{S E}^{(0)}\right)$ given in Eq. (73). Both $T_{\text {orb }}$ and $T_{C}$ should, of course, be reduced by fluctuations (indeed, with $J_{\text {exp }}=-2.75 \mathrm{meV},{ }^{10}$ one obtains mean-field $T_{C} \simeq 48 \mathrm{~K}$ instead of observed $27 \mathrm{~K}$ ), so it makes more sense to consider their ratio, which is

$$
T_{\text {orb }} / T_{C} \simeq 2.2
$$

For $T_{C} \sim 25-30 \mathrm{~K}$ (which is sample dependent), this gives an upper estimation $T_{\text {orb }} \sim 55-70 \mathrm{~K}$. We would like to think that local orbital order, accompanied by short-range ferromagnetic correlations, starts to develop 
at these temperatures. In fact, the presence of such a correlations in $\mathrm{YTiO}_{3}$ below $\sim 50-60 \mathrm{~K}$ has been reported from several experiments: (i) sharp drop in NMR relaxation rate, which has been speculated in terms of orbital ordering, ${ }^{34}$ (ii) spin-resonance line shape changes from typical paramagnetic spectra to the ferromagnetic one, ${ }^{35}$ (iii) weak quasielastic magnetic scattering is observed above $T_{C} \cdot{ }^{10}$

\section{ANGULAR MOMENTUM FLUCTUATIONS: DYNAMICAL MAGNETIC SUSCEPTIBILITY}

In this section, we would like to calculate orbital contribution to the inelastic neutron-scattering intensity. The point is that $t_{2 g}$ orbitals are magnetically active, as their angular momentum may directly couple to the neutrons. Of course, there is a contribution also in nonmagnetic channels: The orbital quadrupole moment is coupled to the phonons, and hence single or double orbiton (depending on the structure of this coupling) may be excited by neutrons indirectly via lattice vibrations. We focus on the magnetic scattering, and calculate orbital angular momentum dynamical susceptibility. If the $t_{2 g}$ orbital level is split up by strong lattice distortions, one would expect just a local, crystal-field transitions. In SE-driven orbital picture, advocated in this paper, angular momentum fluctuations are however of the collective nature. Thus, we expect momentum selected (though strongly damped) transitions, forming broad bands.

\section{A. Quadrupole order}

Consider first local angular momentum susceptibility $\chi_{l o c}(\omega)$ in the quadrupole ordered state. It is defined as

$$
\chi_{l o c}(\omega)=\left\langle\vec{l}_{i} \cdot \vec{l}_{i}\right\rangle,
$$

and its imaginary part describes the spectral shape of the momentum-integrated inelastic neutron-scattering cross section.

In a linear orbital wave approximation, the imaginary part of $\chi_{l o c}(\omega)$ at $\omega>0$ is given by

$$
\chi_{l o c}^{\prime \prime}(\omega)=\pi \sum_{\vec{k}}\left[\frac{1}{\omega_{1 \vec{k}}} \delta\left(\omega-\omega_{1 \vec{k}}\right)+\frac{1}{\omega_{2 \vec{k}}} \delta\left(\omega-\omega_{2 \vec{k}}\right)\right] .
$$

In order to account for a finite gap induced in the orbital sector by lattice distortions (see Sec.V), we use hereafter Eq. (66) for the orbital excitation spectrum. The numerical result for $\chi_{l o c}^{\prime \prime}(\omega)$ is presented in Fig. 12. The sharp structure about $W_{\text {orb }}$ (taken as an energy scale in the figure) is related to the orbiton band-edge effects, which should go away when damping effects are properly taken into account.

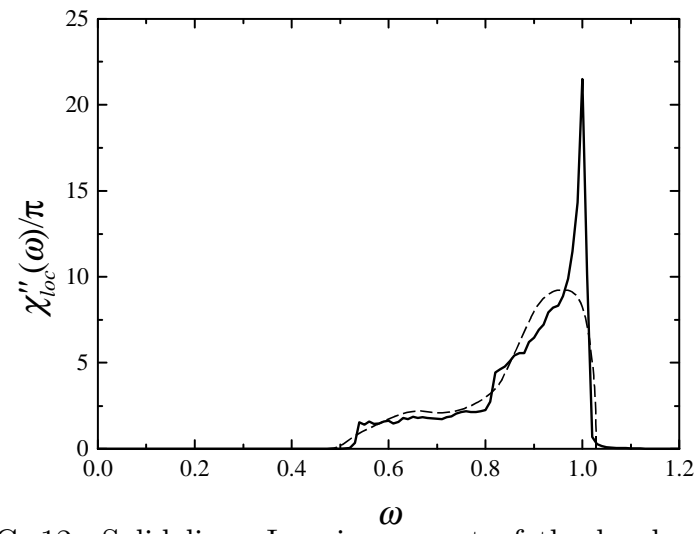

FIG. 12. Solid line: Imaginary part of the local angular momentum susceptibility $\chi_{\text {loc }}^{\prime \prime}(\omega)$ [Eq.(102)] in quadrupole ordered state of orbitals. The energy $\omega$ is given in units of $W_{\text {orb }}$ defined by Eq.(67). A finite gap for the orbital waves stemming from symmetry breaking interactions is taken into account according to Eq. (66) with parameters $f=0.086$ and $\varepsilon=0.18$ (this gives $W_{\text {orb }} \simeq 2 r_{1} J_{S E}$ ). The sharp peak structure is expected to be smoothed by damping effects (not accounted in the present study), as indicated by the broken line.

We turn now to the momentum dependence of dynamical susceptibility, $\chi(\vec{q}, \omega)=\left\langle\vec{l}_{\vec{q}} \cdot \vec{l}_{-\vec{q}}\right\rangle_{\omega}$. This quantity determines a dynamical structure factor, which at $T=0$ is given by

$$
S(\vec{q}, \omega)=\frac{1}{\pi} \operatorname{Im} \chi(\vec{q}, \omega) .
$$

A noncollinear, four sublattice orbital order leads to the following structure for $\chi(\vec{q}, \omega)$ :

$$
\begin{aligned}
\chi(\vec{q}, \omega)= & \frac{2}{3}\left[\chi_{1}\left(\vec{q}+\vec{q}_{1}\right)+\chi_{1}\left(\vec{q}+\vec{q}_{2}\right)+\chi_{1}\left(\vec{q}+\vec{q}_{3}\right)\right]_{\omega} \\
& +\frac{1}{\sqrt{3}}\left[\chi_{2}\left(\vec{q}+\vec{q}_{2}\right)-\chi_{2}\left(\vec{q}+\vec{q}_{1}\right)\right]_{\omega} \\
& +\frac{1}{3}\left[2 \chi_{3}\left(\vec{q}+\vec{q}_{3}\right)-\chi_{3}\left(\vec{q}+\vec{q}_{1}\right)-\chi_{3}\left(\vec{q}+\vec{q}_{2}\right)\right]_{\omega} .
\end{aligned}
$$

Here, the orbital ordering vectors

$$
\begin{aligned}
& \vec{q}_{1}=(\pi, 0, \pi), \\
& \vec{q}_{2}=(\pi, \pi, 0), \\
& \vec{q}_{3}=(0, \pi, \pi)
\end{aligned}
$$

for the state (a) and

$$
\begin{aligned}
& \vec{q}_{1}=(\pi, \pi, \pi), \\
& \overrightarrow{q_{2}}=(\pi, \pi, 0), \\
& \overrightarrow{q_{3}}=(0,0, \pi)
\end{aligned}
$$

for the state (b). The susceptibilities

$$
\chi_{1}(\vec{q}, \omega)=\frac{1}{2}\left\langle\tilde{l}_{\vec{q}}^{x} \tilde{l}_{-\vec{q}}^{x}+\tilde{l}_{\vec{q}}^{y} \tilde{l}_{-\vec{q}}^{y}\right\rangle_{\omega}
$$




$$
\begin{aligned}
\chi_{2}(\vec{q}, \omega) & =\frac{1}{2}\left\langle\tilde{l}_{\vec{q}}^{y} \tilde{l}_{-\vec{q}}^{y}-\tilde{l}_{\vec{q}}^{x} \tilde{l}_{-\vec{q}}^{x}\right\rangle_{\omega}, \\
\chi_{3}(\vec{q}, \omega) & =\frac{1}{2}\left\langle\tilde{l}_{\vec{q}}^{x} \tilde{l}_{-\vec{q}}^{y}+\tilde{l}_{\vec{q}}^{y} \tilde{l}_{-\vec{q}}^{x}\right\rangle_{\omega}
\end{aligned}
$$

are defined in a rotated basis given by the transformation in Eq. (19). We calculate these susceptibilities in a linear orbital-wave approximation. The imaginary part of $\chi_{\alpha}(\vec{q}, \omega)$ at $\omega>0$ is obtained as

$$
\begin{aligned}
\frac{1}{\pi} \chi_{\alpha}^{\prime \prime}(\vec{q}, \omega)= & A_{\alpha} \frac{1+\gamma_{1}+\kappa}{\omega_{1 \vec{q}}} \delta\left(\omega-\omega_{1 \vec{q}}\right) \\
& +B_{\alpha} \frac{1+\gamma_{1}-\kappa}{\omega_{2 \vec{q}}} \delta\left(\omega-\omega_{2 \vec{q}}\right),
\end{aligned}
$$

where $A_{1}=B_{1}=1 / 2, A_{2}=-B_{2}=\gamma_{2} / 2 \kappa$ and $A_{3}=$ $-B_{3}=\gamma_{3} / 2 \kappa$.

Numerical results for $S(\vec{q}, \omega)$ in the state I (a) are shown in Fig. 13. An intensive hot spot at momentum $\vec{q}=(\pi, \pi, \pi)$ at energies about orbiton gap, and flat dispersions at $\left(\pi, \pi, q_{z}\right)$ direction are noticed. The rather complicated multiband structure has its origin in noncollinear nature of the underlying orbital ordering, characterized by a several ordering vectors. [In the state I (b), which has different ordering vectors $\vec{q}_{i}, S(\vec{q}, \omega)$ shows different energy-momentum structure (not shown)]. The energy scale for orbital fluctuations $\left(\sim W_{\text {orb }} \sim 2 r_{1} J_{S E}\right)$ is much larger than magnon energies. This is because of strong cancellation of ferromagnetic and $\mathrm{AF}$ contributions to the spin-exchange integral $J$ (see Sec. VI A), resulting in rather small magnon bandwidth (which is only a fraction of $r_{1} J_{S E}$ ). Therefore, magnon excitations are expected to be well defined, since they are located within the orbital gap. As for the high-energy orbital excitations, we expect strong damping effects stemming from nonlinear couplings between orbital waves themselves, and also from the dynamical coupling between spin and orbital fluctuations. These effects should in fact relax momentum resolution and smooth away sharp structures obtained in Fig. 13 by using undamped orbital waves.

\section{B. Orbital magnetic state}

For completeness, we also give equations for the magnetic response of the magnetically ordered state of orbitals. In contrast to the quadrupole ordering, this state gives rise to static Bragg peaks of orbital origin. These peaks are located at orbital ordering vectors $\vec{q}_{i}$ [Eqs.(105) and (106)]:

$$
\left\langle\vec{l}_{\vec{q}} \cdot \vec{l}_{-\vec{q}}\right\rangle=\frac{1}{3}\left\langle m_{l}\right\rangle^{2}\left[\delta\left(\vec{q}-\vec{q}_{1}\right)+\delta\left(\vec{q}-\vec{q}_{2}\right)+\delta\left(\vec{q}-\vec{q}_{3}\right)\right],
$$

and their intensity is determined by the orbital magnetic order parameter $m_{l}=0.19$ [Eq.(38)].

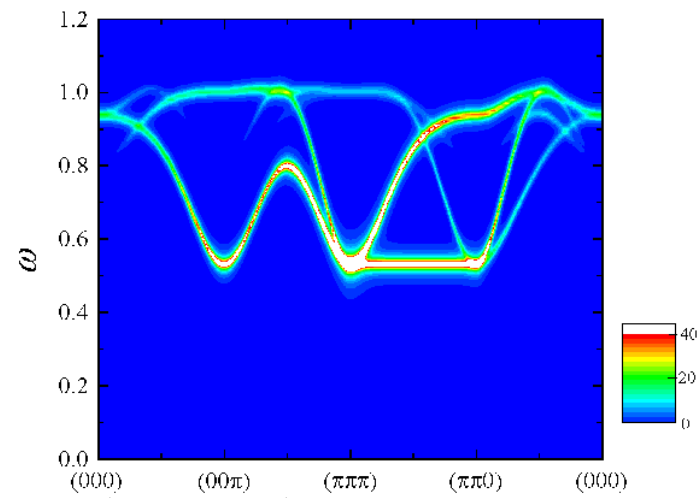

FIG. 13. (Color online). Intensity of the orbital contribution to the magnetic structure factor $S(\vec{q}, \omega)$ [Eq.(103)] in the quadrupole ordered state I(a). Energy $\omega$ is given in units of $W_{\text {orb }}$ defined by Eq.(67). A finite orbital excitation gap due to symmetry breaking terms (see for details Sec.V) is taken into account according to Eq. (66) with parameters $f=0.086$ and $\varepsilon=0.18$.

The dynamical susceptibility $\chi(\vec{q}, \omega)$ is given by the same form as Eq. (104) with $\chi_{1,2,3}(\vec{q}, \omega)$ having the same definitions given by Eqs. (107). In the orbital magnetic state, these susceptibilities are obtained as follows:

$$
\chi_{1}^{\prime \prime}(\vec{q}, \omega)=\frac{\pi}{4}\left[\frac{1}{\omega_{1 \vec{q}}} \delta\left(\omega-\omega_{1 \vec{q}}\right)+\frac{1}{\omega_{2 \vec{q}}} \delta\left(\omega-\omega_{2 \vec{q}}\right)\right]
$$

and

$$
\chi_{2,3}^{\prime \prime}(\vec{q}, \omega)=\frac{\pi \gamma_{2,3}}{4 \kappa}\left[\frac{\gamma_{1}+\kappa}{\omega_{1 \vec{q}}} \delta\left(\omega-\omega_{1 \vec{q}}\right)-\frac{\gamma_{1}-\kappa}{\omega_{2 \vec{q}}} \delta\left(\omega-\omega_{2 \vec{q}}\right)\right] .
$$

\section{SUMMARY AND DISCUSSION}

In this paper we investigated a spin-orbital superexchange Hamiltonian in a Mott insulator with $t_{2 g}^{1}$ electron configuration, focusing mainly on the orbital order and dynamics in the spin ferromagnetic state. An important feature of the Hamiltonian in the spin polarized state is the large frustration of orbital states, thus the ground state is governed by the interplay between orbital frustration and quantum fluctuations. On the classical level, there is a local $Z_{2}$ symmetry which leads to an infinite degeneracy of classical configurations. Longrange orbital order does occur in the model by a quantum order-from-disorder mechanism, which selects a particular ordering patterns. Orbital orderings are quite unusual having highly noncollinear four sublattice structure, and provide the same spin couplings in all cubic directions.

Besides classical local $Z_{2}$ symmetry which is removed by quantum dynamics, there are exact conservation laws in the orbital Hamiltonian. They are related to the conservation of orbital quantum numbers in the SE process, and lead to a multitude of degenerate quantum ground 
states which can smoothly be connected to each other by phase rotations of the complex orbital order parameter. Such continuous rotations generate orbital Goldstone modes, which have 2D dispersion because of planar geometry of $t_{2 g}$ orbitals. As a result, static orbital order sets in at zero temperature only. Degenerate quantum ground states are physically different: depending on the phase of the orbital condensate, they describe quadrupole or magnetic orderings or their coherent mixture. Extrinsic perturbations, e.g. lattice distortions or spin-orbit interactions may remove the degeneracy and fix the phase of the condensate. Reflecting the large quantum fluctuations, the orbital order parameter is unusually small.

We found that the orbitally ordered ferromagnetic state is slightly higher in energy than the spin-AF orbital liquid state. This is because the latter state gains an additional quantum energy from coupled spin-orbital fluctuations. To explain ferromagnetism of $\mathrm{YTiO}_{3}$, we emphasized the role played by Ti-O-Ti bond angle distortion. This distortion favors ferrostate by generating an unfrustrated ferromagnetic SE interaction via virtual hopping of electrons between $\mathrm{NN} t_{2 g}$ and $e_{g}$ orbitals. Even more importantly, the bond distortion eliminates orbital soft modes and opens a large orbital gap, such that orbital order becomes stable at finite temperature. This distortion stabilizes the quadrupole ordered state.

The strong competition between AF to $\mathrm{F}$ states in the present model has direct relevance to nearly continuous transition between these states observed in titanates. In these compounds, $A$-site substitution from La to Y increases the Ti-O-Ti bond distortion, hence changing gradually a delicate balance between $\mathrm{AF}$ and $\mathrm{F}$ states. Because of the orbital fluctuations, spin-exchange integral on every link experiences strong fluctuations, both in amplitude and in sign, and the system may develop either an AF or F state depending on local orbital correlations. In this picture of "fluctuating exchange bonds," the magnetic transition temperatures, $T_{N}$ and $T_{C}$, represent only a time-averaged static component of the spin couplings. Its value is only a fraction of full superexchange energy scale, and can gradually be tuned by external forces such as lattice distortion, pressure, etc. We think that weak orbital order may continuously evolve in titanates when the bond angle decreases below a certain critical value, and propose the phase diagram shown in Fig. 14. The sign of the time-averaged spin coupling depends on a local correlation of orbitals. To the right of the critical point orbital correlations are more antiferromagnetic, supported by noncollinear orbital orderings. To the left, the genuine ground state of the $t_{2 g}$ superexchange, an orbital disordered state supporting spin AF is stabilized. In the proximity area, a fluctuating part of the overall superexchange interaction dominates, and separation of the spin and orbital degrees of freedom might no longer be possible. This scenario can be tested experimentally by investigating the spin and orbital transition temperatures under high pressure and magnetic field. On the theoretical side, a quantitative description of the transi-

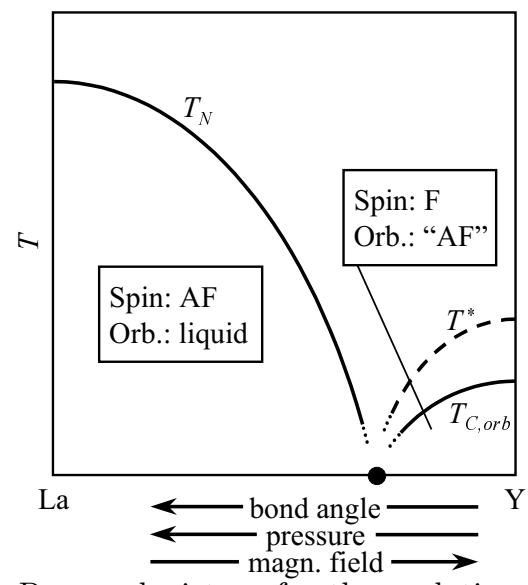

FIG. 14. Proposed picture for the evolution of magnetic and orbital states in perovskite titanates. $T_{C}$ and $T_{N}$ are Curie and Néel spin ordering temperatures, respectively. Orbital ordering below $T_{\text {orb }} \simeq T_{C}$ is expected in ferromagnetic region. Below $T^{*}$, short range ferro-type spin and noncollinear, dominantly "AF"-type orbital correlations grow up.

tion between ferromagnetic and AF states, controlled by orbital order-disorder transition, remains an interesting and challenging problem.

Further, in order to discuss the recent spin-wave data in $\mathrm{YTiO}_{3}$, we derived the low-energy spin Hamiltonian by including a relativistic spin-orbit coupling and lattice distortions that induce the orbiton gap. Using this Hamiltonian, we calculated the magnon gap and found that it is very small because of high symmetry of the underlying orbital order. Both the real and the spin space isotropy of the spin-wave spectra observed experimentally find natural explanations within the proposed theory.

We also calculated the orbital contribution to resonant $\mathrm{x}$-ray intensity and to dynamical magnetic structure factor. Predictions made should be helpful in further experimental study of titanates.

\section{ACKNOWLEDGMENTS}

We would like to thank B. Keimer and S. Maekawa for stimulating discussions. Discussions with C. Ulrich, S. Ishihara, T. Kiyama, M. Itoh and J. Akimitsu are also acknowledged. One of us (G. Kh.) would like to thank International Frontier Center for Advanced Materials at the Institute for Materials Research, Tohoku University, where a part of this work was carried out, for its kind hospitality. S. O. acknowledges the hospitality of MaxPlanck-Institut für Festkörperforschung in Stuttgart during his several visits. 


\section{APPENDIX A: BOND DEPENDENCE OF QUADRUPOLE MOMENT OPERATORS}

In this appendix, we present the explicit expressions for $Q_{\alpha}^{(\gamma)}, T_{\alpha}^{(\gamma)}$. By replacing $l_{\alpha}$ in Eqs. (24) with $l_{\alpha}^{(\gamma)}$, one obtains

$$
\begin{aligned}
Q_{x}^{(\gamma)} & =\left\{\begin{aligned}
-c Q_{x} \mp s T_{z}, & \text { for } \gamma=a(b) \\
Q_{x}, & \text { for } \gamma=c
\end{aligned}\right. \\
T_{z}^{(\gamma)} & =\left\{\begin{aligned}
-c T_{z} \pm s Q_{x}, & \text { for } \gamma=a(b) \\
T_{z}, & \text { for } \gamma=c
\end{aligned}\right. \\
T_{x}^{(\gamma)} & =\left\{\begin{aligned}
-c T_{x} \mp s T_{y}, & \text { for } \gamma=a(b) \\
T_{x}, & \text { for } \gamma=c
\end{aligned}\right. \\
T_{y}^{(\gamma)} & =\left\{\begin{aligned}
-c T_{y} \pm s T_{x}, & \text { for } \gamma=a(b) \\
T_{y}, & \text { for } \gamma=c
\end{aligned}\right.
\end{aligned}
$$

$T_{ \pm 1}^{(\gamma)}$ is given by $T_{ \pm 1}^{(\gamma)}=T_{y}^{(\gamma)} \pm T_{x}^{(\gamma)}$.

\section{APPENDIX B: COEFFICIENTS OF BOGOLIUBOV TRANSFORMATION}

We present the coefficients of Bogoliubov transformation which diagonalize $H_{O W}$ in Eq. (28). This transformation reads as follows:

$$
\begin{aligned}
\tilde{a}_{\vec{k}}= & u \cosh \theta_{1} \alpha_{1 \vec{k}}+v \cosh \theta_{2} \alpha_{2 \vec{k}}-u \sinh \theta_{1} \alpha_{1-\vec{k}}^{\dagger} \\
& -v \sinh \theta_{2} \alpha_{2-\vec{k}}^{\dagger}, \\
\tilde{b}_{\vec{k}}= & -v \cosh \theta_{1} \alpha_{1 \vec{k}}+u \cosh \theta_{2} \alpha_{2 \vec{k}}+v \sinh \theta_{1} \alpha_{1-\vec{k}}^{\dagger} \\
& -u \sinh \theta_{2} \alpha_{2-\vec{k}}^{\dagger} .
\end{aligned}
$$

The inverse transformation is given as follows:

$$
\begin{aligned}
\alpha_{1 \vec{k}}= & u \cosh \theta_{1} \tilde{a}_{\vec{k}}-v \cosh \theta_{1} \tilde{b}_{\vec{k}}+u \sinh \theta_{1} \tilde{a}_{-\vec{k}}^{\dagger} \\
& -v \sinh \theta_{1} \tilde{b}_{-\vec{k}}^{\dagger}, \\
\alpha_{2 \vec{k}}= & v \cosh \theta_{2} \tilde{a}_{\vec{k}}+u \cosh \theta_{2} \tilde{b}_{\vec{k}}+v \sinh \theta_{2} \tilde{a}_{-\vec{k}}^{\dagger} \\
& +u \sinh \theta_{2} \tilde{b}_{-\vec{k}}^{\dagger} .
\end{aligned}
$$

Here, $u$ and $v$ are

$$
u=\frac{1}{\sqrt{2}} \sqrt{1+\frac{\gamma_{2}}{\kappa}}
$$

and

$$
v=\frac{1}{\sqrt{2}} \sqrt{1-\frac{\gamma_{2}}{\kappa}} \operatorname{sgn}\left(\gamma_{3}\right),
$$

respectively, and $\theta_{1,2}$ is given by $\tanh 2 \theta_{1,2}=\gamma_{1} \pm \kappa$.

\section{APPENDIX C: MAGNON GAP BY SINGLE-MODE APPROXIMATION}

We examine here the magnon excitation gap, generated by "cubic" anisotropy interaction given in Sec. VIB, by using a different approach: namely, we apply single-mode approximation. ${ }^{29}$ In this approximation, spin excitation energy at $\vec{q} \rightarrow 0$ is given as follows:

$$
\omega_{\vec{q} \rightarrow 0}=\frac{\left\langle\left[\left[S_{\vec{q}}^{+}, H_{s}\right], S_{-\vec{q}}^{-}\right]\right\rangle_{\vec{q} \rightarrow 0}}{\left\langle S_{\vec{q}}^{+} S_{-\vec{q}}^{-}\right\rangle_{\vec{q} \rightarrow 0}},
$$

where $H_{s}$ is given in Eq. (89) with $S_{i}^{(a)}=S_{i}^{x}, S_{i}^{(b)}=S_{i}^{y}$, and $S_{i}^{(c)}=S_{i}^{z}$. The double-commutator correlation function in the numerator of Eq. (C1) is equal to $\sum_{i j} \Gamma_{i j}^{(\gamma)}$, where

$$
\begin{aligned}
& \Gamma_{i j}^{(a)}=-\tilde{A}\left\langle S_{i}^{z} S_{j}^{z}-\frac{1}{2}\left(S_{i}^{-} S_{j}^{+}+S_{i}^{-} S_{j}^{-}\right)\right\rangle, \\
& \Gamma_{i j}^{(b)}=-\tilde{A}\left\langle S_{i}^{z} S_{j}^{z}-\frac{1}{2}\left(S_{i}^{-} S_{j}^{+}-S_{i}^{-} S_{j}^{-}\right)\right\rangle, \\
& \Gamma_{i j}^{(c)}=-\tilde{A}\left\langle-2 S_{i}^{z} S_{j}^{z}+S_{i}^{+} S_{j}^{-}\right\rangle .
\end{aligned}
$$

By using linear spin-wave theory, one obtains

$$
\begin{aligned}
\sum_{\langle i j\rangle} \Gamma_{i j}^{(\gamma)} & =2 z S \tilde{A} \sum_{\vec{k}}\left[\gamma_{3}\left\langle a_{\vec{k}}^{\dagger} a_{\vec{k}}\right\rangle-\frac{1}{\sqrt{3}} \gamma_{2}\left\langle a_{\vec{k}}^{\dagger} a_{-\vec{k}}^{\dagger}\right\rangle\right], \\
\left\langle S_{\vec{q}}^{+} S_{-\vec{q}}^{-}\right\rangle_{\vec{q} \rightarrow 0} & =2 S\left\langle 1+a_{0}^{\dagger} a_{0}\right\rangle .
\end{aligned}
$$

Expectation values $\left\langle a_{\vec{k}}^{\dagger} a_{\vec{k}}\right\rangle$ and $\left\langle a_{\vec{k}}^{\dagger} a_{-\vec{k}}^{\dagger}\right\rangle$ are calculated at $T=0$. Up to linear in $\tilde{A}$ terms, one obtains $\left\langle a_{\vec{k}}^{\dagger} a_{\vec{k}}\right\rangle=0$ and

$$
\left\langle a_{\vec{k}}^{\dagger} a_{-\vec{k}}^{\dagger}\right\rangle=\frac{\tilde{A}}{2 z J} \frac{c_{x}-c_{y}}{1-\gamma_{1}} .
$$

Consequently, we find the magnon gap

$$
\omega_{\vec{q} \rightarrow 0}=\frac{2 \tilde{A}^{2} R}{z J},
$$

where $R$ is given by Eq. (93). This is exactly the result obtained in Sec. VIC.

\section{APPENDIX D: EFFECT OF ORBITAL EXCITATIONS ON THE MAGNON DISPERSION}

In Sec. VIA, we discussed the renormalization of nearest-neighbor isotropic spin coupling $J$ by orbital fluctuations. Here, we investigate this effect in more detail, by considering effects of the dynamical spin/orbital coupling on magnon spectra. In terms of magnon $s_{\vec{p}}$ and 
orbiton $\tilde{a}_{\vec{q}}, \tilde{b}_{\vec{q}}$ operators, the dynamical spin/orbital coupling in Eq.(69) is expressed as (in units of $r_{1} J_{S E}$ )

$$
\begin{gathered}
H_{\text {int }}=-\frac{1}{2} \sum_{\vec{p} \vec{q} \vec{q}^{\prime}} s_{\vec{p}^{\prime}}^{\dagger} s_{\vec{p}}\left[Q \Gamma_{0}\left(\tilde{a}_{\vec{q}^{\prime}}^{\dagger} \tilde{a}_{-\vec{q}}+\tilde{b}_{\vec{q}^{\prime}}^{\dagger} \tilde{b}_{-\vec{q}}\right)\right. \\
+\left(\Gamma_{1}+\Gamma_{2}\right) \tilde{a}_{\vec{q}^{\prime}}^{\dagger} \tilde{a}_{\vec{q}}^{\dagger}+\left(\Gamma_{1}-\Gamma_{2}\right) \tilde{b}_{\vec{q}^{b}}^{\dagger} \tilde{b}_{\vec{q}}^{\dagger} \\
\left.-2 \Gamma_{3} \tilde{a}_{\vec{q}^{\prime}}^{\dagger} \tilde{b}_{\vec{q}}^{\dagger}\right]+H . c .
\end{gathered}
$$

where $\vec{p}^{\prime}=\vec{p}-\vec{q}-\vec{q}^{\prime}$. Factor $Q \simeq 0.19$ stems from Hartree decoupling of the $Q_{i z} Q_{j z}$ term in Eq. (23). $\Gamma_{0}$ and $\Gamma_{i(=1,2,3)}$ are given as follows:

$$
\begin{aligned}
\Gamma_{0} & =1+\gamma_{1 \vec{q}+\vec{q}^{\prime}}-\gamma_{1 \vec{p}}-\gamma_{1 \vec{q}+\vec{q}^{\prime}-\vec{p}} \\
\Gamma_{i} & =\gamma_{i \vec{q}}+\gamma_{i \vec{q}^{\prime}}-\gamma_{i \vec{q}-\vec{p}}-\gamma_{i \vec{q}^{\prime}-\vec{p}} .
\end{aligned}
$$

Second-order perturbation with respect to $H_{\text {int }}$ gives the renormalization of magnon excitation energy as $\omega_{\vec{p}}=$ $\omega_{\vec{p}}^{(0)}-\delta \omega_{\vec{p}}$. Here, magnon softening $\delta \omega_{\vec{p}}$ is given by

$$
\begin{aligned}
\delta \omega_{\vec{p}}=\frac{1}{2} \sum_{\vec{q} \vec{q}^{\prime}} & {\left[\frac{M(1+\lambda)}{\omega_{1 \vec{q}}+\omega_{1 \vec{q}^{\prime}}+\omega_{\vec{p}^{\prime}}-\omega_{\vec{p}}}\right.} \\
& \left.+\frac{\widetilde{M}(1+\tilde{\lambda})}{\omega_{1 \vec{q}}+\omega_{2 \vec{q}^{\prime}}+\omega_{\vec{p}^{\prime}}-\omega_{\vec{p}}}\right],
\end{aligned}
$$

where $\omega_{1,2 \vec{q}}=\left(\mu^{2}-\gamma_{ \pm}^{2}\right)^{1 / 2}$ with $\gamma_{ \pm}=\gamma_{1} \pm \kappa$ correspond to two orbiton branches. The orbiton chemical potential $\mu$ controls the orbital gap. Magnon energy $\omega_{p}$ that enters in this equation is considered to have a NN Heisenberg form $3|J|\left(1-\gamma_{1 \vec{q}}\right)$. The matrix elements in the numerator have a following structure:

$$
\begin{array}{r}
M=\left(\Gamma_{1}+r \Gamma_{0}\right)^{2}(1+x)+\Gamma_{2}^{2}(1+y)+\Gamma_{3}^{2}(1-y) \\
+2\left(\Gamma_{1}+r \Gamma_{0}\right)\left(\Gamma_{2} c_{2}+\Gamma_{3} c_{3}\right)+2 \Gamma_{2} \Gamma_{3} z,
\end{array}
$$

where

$$
\begin{aligned}
\lambda & =\frac{\mu^{2}+\gamma_{+\vec{q}} \gamma_{+\vec{q}^{\prime}}-\omega_{1 \vec{q}} \omega_{1 \vec{q}^{\prime}}}{2 \omega_{1 \vec{q}} \omega_{1 \vec{q}^{\prime}}}, \\
r & =-\frac{\mu}{1+\lambda} \frac{\gamma_{+\vec{q}}+\gamma_{+\vec{q}^{\prime}}}{2 \omega_{1 \vec{q}^{2}} \omega_{1 \vec{q}^{\prime}}} Q, \\
x & =\left(\gamma_{2 \vec{q}} \gamma_{2} \vec{q}^{\prime}+\gamma_{3 \vec{q}} \gamma_{3 \vec{q}^{\prime}}\right) / \kappa_{\vec{q}} \kappa_{\vec{q}^{\prime}}, \\
y & =\left(\gamma_{2 \vec{q}} \gamma_{2 \vec{q}^{\prime}}-\gamma_{3 \vec{q}} \gamma_{3 \vec{q}^{\prime}}\right) / \kappa_{\vec{q}} \kappa_{\vec{q}^{\prime}}, \\
z & =\left(\gamma_{2 \vec{q}} \gamma_{3 \vec{q}^{\prime}}+\gamma_{3 \vec{q}} \gamma_{2 \vec{q}^{\prime}}\right) / \kappa_{\vec{q}} \kappa_{\vec{q}^{\prime}},
\end{aligned}
$$

and

$$
\begin{aligned}
& c_{2}=\frac{\gamma_{2 \vec{q}}}{\kappa_{\vec{q}}}+\frac{\gamma_{2 \vec{q}^{\prime}}}{\kappa_{\vec{q}^{\prime}}}, \\
& c_{3}=\frac{\gamma_{3 \vec{q}}}{\kappa_{\vec{q}}}+\frac{\gamma_{3 \vec{q}^{\prime}}}{\kappa_{\vec{q}^{\prime}}} .
\end{aligned}
$$

Interband orbiton transitions are represented by a second term in Eq. (D3). To obtain $\widetilde{M}$ and $\tilde{\lambda}$, one should just replace $\kappa_{\vec{q}^{\prime}} \rightarrow-\kappa_{\vec{q}^{\prime}}$ in above equations. (This also

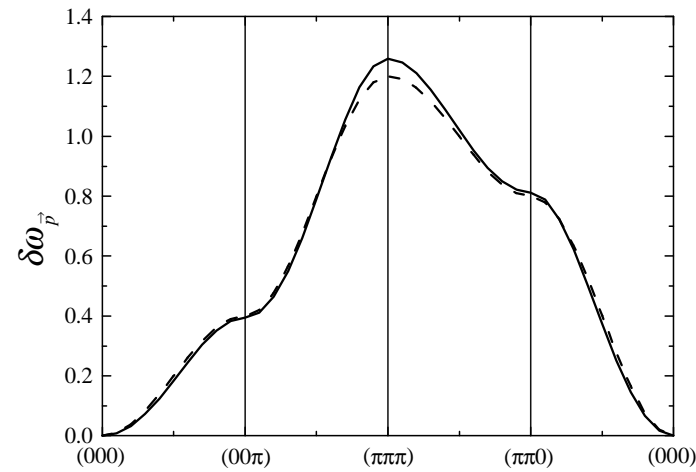

FIG. 15. Solid line: The reduction of the magnon energy $\delta \omega_{p}$ (in units of $r_{1} J_{S E}$ ), calculated from Eq.(D3). Broken line is a function $3(\delta J)\left(1-\gamma_{1} \vec{p}\right)$ with $\delta J=0.2$, showing that the effect of $\delta \omega_{p}$ can fairly be regarded as an effective reduction of NN spin coupling.

leads to $\omega_{1 \vec{q}^{\prime}} \rightarrow \omega_{2} \vec{q}^{\prime}$ in $\lambda$ and $r$ ). One can verify that the function $\delta \omega_{\vec{p}}$ has a cubic symmetry in a momentum space. This property is guaranteed by the high symmetry of the underlying orbital order in any level of approximations. The longer-range, next-NN spin couplings might, of course, be dynamically generated by orbital fluctuations.

We show the numerical result for magnon renormalization $\delta \omega_{\vec{p}}$ in Fig. 15. For magnon dispersion in Eq.(D3) we used $|J|=0.03\left(r_{1} J_{S E}^{(0)}\right) \simeq 0.05\left(r_{1} J_{S E}\right)$ as obtained from Eq. (73), while orbiton dispersion is calculated with $\mu=1.41$ which gives orbiton gap $\simeq r_{1} J_{S E}$. As estimated in the main text, such a gap would be induced by nondiagonal hopping To see a deviation of the magnon remormalization $\delta \omega$ from the NN Heisenberg form, broken line shows a function $3(\delta J)\left(1-\gamma_{1 \vec{q}}\right)$ with $\delta J=0.2$. It is noticed that $\delta J$ is indeed close to $\delta J_{\text {eff }}=2 / 9$ obtained in the main text for the reduction of NN spin couplings. Slight deviations from simple NN model are however visible, in particular a stronger softening at the $(\pi \pi \pi)$ point is seen. By a numerical fitting, these deviations can be traced back to the appearance of longer-range ferromagnetic couplings $J_{2}=-0.003, J_{3}=0$, and $J_{4}=-0.007$ (all in units of $r_{1} J_{S E}$ ). This is understood due to longerrange orbital singlet correlations along the cubic directions, as $J_{4}$ corresponds to a ferromagnetic coupling between second-nearest-neighbor spins along cubic axes. In principle, these corrections could be observable as a slight enhancement of the ratio of small momentum spin stiffness to full magnon bandwidth as compare to the NN Heisenberg model. We should notice however that more quantitative predictions are not possible at the present stage of the theory for rather obvious reason: A dynamical spin-orbital interaction is strong (with the coupling constant being of the order of 1 ), so a more elaborate treatment is needed to quantify the strongly correlated model under consideration. 


\section{APPENDIX E: EXAMINATION OF SPIN INTERACTIONS IN THE PREVIOUS ORBITAL MODELS FOR YTIO 3}

In this appendix, we examine the magnetic interactions in the orbital state previously reported in Refs. 15, 23-26, in order to check whether this state can explain recent neutron-scattering results on the spin couplings, spin canting, and magnon gap.

The Heisenberg spin exchange coupling: First, we discuss bond dependence of the isotropic spin interactions. The orbital state reported by Hartree-Fock ${ }^{15}$ and bandstructure $^{23}$ calculations are expressed as

$$
\begin{array}{r}
\left|\psi_{1,3}\right\rangle=\sqrt{n_{c}}\left|d_{x y}\right\rangle \pm \sqrt{1-n_{c}}\left|d_{x z}\right\rangle \\
\left|\psi_{2,4}\right\rangle=\sqrt{n_{c}}\left|d_{x y}\right\rangle \pm \sqrt{1-n_{c}}\left|d_{y z}\right\rangle
\end{array}
$$

with $n_{c}$ being an occupation of the $x y$ orbital. Using these wave functions, it is easy to obtain from Eq. (2) spin exchange couplings along $c$-, $a$ and $b$-axes:

$J^{(c)}=\frac{1}{2} J_{S E}\left[\left(r_{1}+r_{2} r_{3}\right)\left(1-n_{c}\right)-\left(r_{1}-r_{2}\right)\right]\left(1-n_{c}\right)$

and

$J^{(a b)}=\frac{1}{2} J_{S E}\left[\left(r_{1}+r_{2} r_{3}\right) n_{c}^{2}-\frac{1}{2}\left(r_{1}-r_{2}\right)\left(1+n_{c}\right)\right]$.

The exchange interactions are presented as functions of $\eta$ in Fig. 16 for different values of $n_{c}$. The "meeting" points, where $J^{(c)}=J^{(a b)}$, are shown by circles for each $n_{c}$. One finds that the isotropy point for the state with $n_{c}=0.5$ (suggested in Refs. 15,23 ) is right at the border $\eta=0$, but the exchange coupling is of the AF sign there. For larger $n_{c}$ one may obtain the isotropy point with $\mathrm{F}$ coupling, but this requires too large values of $\eta$. Moreover, the "meeting" point is extremely sensitive to both $\eta$ and $n_{c}$ and requires fine tuning. As shown in the inset of Fig. 16, the $J^{(a b)} / J^{(c)}$ ratio drastically changes even at a small (just within $\pm 5 \%$ ) variation of $n_{c}$, and may even reverse the sign.

Spin anisotropy interactions: Next, we investigate the effects of spin anisotropy interactions in the state, Eq. (E1). We denote the occupied orbital state described by wave functions in Eq. (E1) by $\alpha$, while the lowest unoccupied state is called $\beta$. The state $\beta$ has wave function which is a counterpart of that for $\alpha$ state; e.g., $\left|\tilde{\psi}_{1}\right\rangle=\sqrt{1-n_{c}}\left|d_{x y}\right\rangle-\sqrt{n_{c}}\left|d_{x z}\right\rangle$ on site 1 . Level separation between $\alpha$ and $\beta$ is introduced as $\Delta_{\alpha \beta}$. Active components of the angular momenta at sites 1, 3 and 2, 4 are $l_{x}$ and $l_{y}$, respectively. These are expressed in terms of orbital doublet operators as follows:

$$
\left(l_{x}\right)_{1,3}= \pm i\left(\beta^{\dagger} \alpha-\alpha^{\dagger} \beta\right)
$$

and

$$
\left(l_{y}\right)_{2,4}=\mp i\left(\beta^{\dagger} \alpha-\alpha^{\dagger} \beta\right) \text {. }
$$

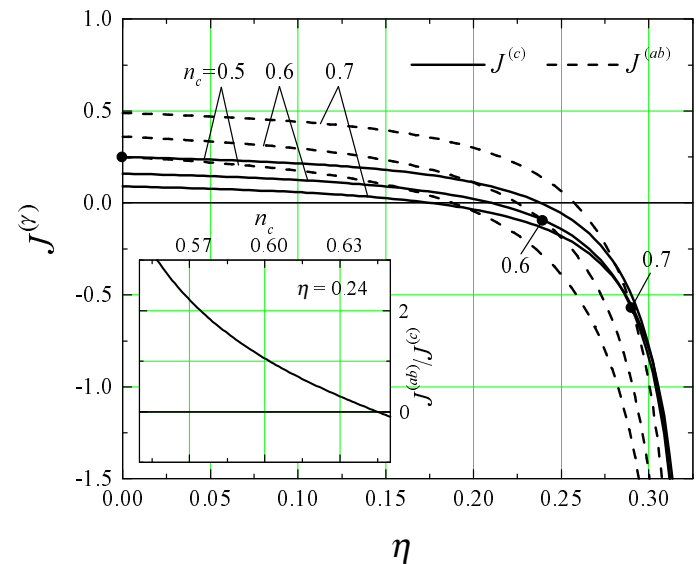

FIG. 16. Spin exchange interactions in the model (E1) as a function of the Hund's coupling $\eta$ for different values of the $x y$-orbital occupation $n_{c}$. Energy unit is $J_{S E}=4 t^{2} / U$. Filled circles show the position where spin couplings are isotropic for fixed value of $n_{c}$. Inset: The ratio $J^{(a b)} / J^{(c)}$ as a function of $n_{c}$ for fixed $\eta=0.24$.

As usual, the spin anisotropy Hamiltonian follows from perturbation theory with respect to spin-orbit coupling $H_{s o}$ and superexchange interaction $H_{S E}$. In the SE operators $\hat{J}_{i j}^{(c)}$ and $\hat{K}_{i j}^{(c)}$, the following terms contribute to the spin anisotropy Hamiltonian: (i) the terms operating in the $\beta$ excited states, and (ii) the terms connecting a ground state $\alpha$ with excited ones $\beta$.

Symmetric spin anisotropy interaction: Third-order perturbation with respect to $H_{s o}$ and $H_{S E}$ gives symmetric spin anisotropy Hamiltonian $H_{a n i}$. For 1-3 bond along the $c$-direction, $H_{a n i}$ is given by

$$
H_{\text {ani }}^{(13)}=\widetilde{A}\left(S_{1 x} S_{3 x}\right)
$$

where

$$
\widetilde{A}=J_{S E} \frac{r_{1}+r_{2}}{4}\left(\frac{\lambda}{\Delta_{\alpha \beta}}\right)^{2} 4 n_{c}\left(1-n_{c}\right) .
$$

Here, the terms proportional to small numbers $\left(r_{1}-r_{2}\right)$ and $\left(r_{2}-r_{3}\right)$ are neglected. For $2-4$ bond along $c$-direction

$$
H_{\text {ani }}^{(24)}=\widetilde{A}\left(S_{2 y} S_{4 y}\right) .
$$

Note that spin components correspond to that of active angular momentum and the interaction is of the AF sign. This originates from the fluctuation of active angular momenta with AF correlation between NN sites, as can be inferred from Eqs. (E4) and (E5). For the 1-2 bond in the $a b$-plane, $H_{a n i}$ has the following form:

$$
H_{\text {ani }}^{(12)}=-\frac{1}{4} \widetilde{A}\left(S_{1 z} S_{2 z}\right)+\frac{1}{2} \widetilde{A}\left(S_{1 y} S_{2 x}\right) .
$$

The combination of spin components in the last term is different from that of active momenta at sites 1 and 2. Such terms originate from the following processes (combination of operators): $\left\langle l_{1 x} S_{1 x}\left(S_{1 z} S_{2 z}\right) l_{2 y} S_{2 y}\right\rangle_{\text {orb }}$, 


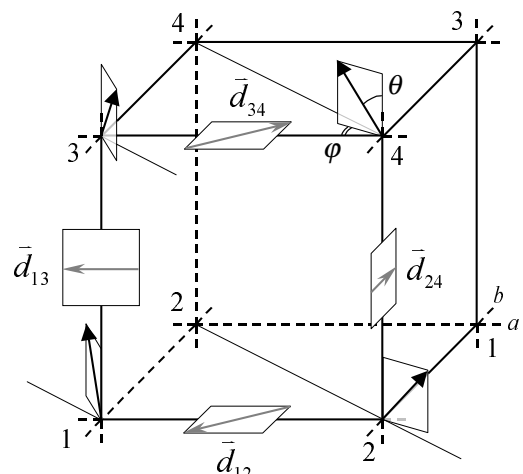

FIG. 17. Antisymmetric DM interaction pattern obtained for the orbital model (E1). Gray arrow denoted by $\vec{d}_{i j}$ shows the orientation of DM vectors. Black arrows represent the direction of spins favorable for these interactions.

$\left\langle l_{2 y} S_{2 y}\left(S_{1 z} S_{2 z}\right) l_{1 x} S_{1 x}\right\rangle_{\text {orb }}$, etc. For $3-4$ bond, symmetric spin anisotropy Hamiltonian $H_{a n i}$ has the same form as $H_{a n i}^{(12)}$, where site index $1(2)$ is replaced with 3 (4). It can be shown that $H_{a n i}$ does not cause finite spin canting when $|J| \gtrsim \widetilde{A} / 2$, where $J$ is the isotropic spin coupling. However, it leads to a large magnon gap of classical origin, which we obtained to be $\sqrt{3} \widetilde{A} S$.

$D M$ interaction: For the 1-3 bond, second-order perturbation with respect to $H_{s o}$ and $H_{S E}$ gives

$$
H_{D M}^{(13)}=\widetilde{D}^{(c)} \vec{d}_{13} \cdot\left(\vec{S}_{1} \times \vec{S}_{3}\right)
$$

where constant $\widetilde{D}^{(c)}$ is obtained as

$$
\widetilde{D}^{(c)}=J_{S E} \frac{r_{1}+r_{2}}{4} \frac{\lambda}{\Delta_{\alpha \beta}} 4 n_{c}^{1 / 2}\left(1-n_{c}\right)^{3 / 2} .
$$

DM vector $\vec{d}_{13}$ is given as $\vec{d}_{13}=(-1,0,0)$. We point out here that DM constant $\widetilde{D}^{(c)}$ is much larger than $D$ in Eq. (87), obtained in the main text for the SEdriven orbital states. The reason is that the orbital order given by Eq.(E1) has not that high symmetry, and the terms proportional to $\left(r_{1}+r_{2}\right)$ in the operator $\hat{J}_{i j}^{(c)}$ do contribute to DM interaction. Therefore, the ratio $\widetilde{D}^{(c)} / D \propto \frac{r_{1}+r_{2}}{r_{1}-r_{2}} \propto 1 / \eta$ is large.

On the in-plane bond 1-2, we find

$$
H_{D M}^{(12)}=\frac{1}{2} \widetilde{D}^{(a b)} \vec{d}_{12} \cdot\left(\vec{S}_{1} \times \vec{S}_{2}\right),
$$

with $\widetilde{D}^{(a b)}=\widetilde{D}^{(c)} n_{c} /\left(1-n_{c}\right)$ and $\vec{d}_{12}=(-1,-1,0)$. The DM interactions on the 2-4 and 3-4 bonds are given by the same forms as Eqs. (E10) and (E12), respectively, where $\vec{d}_{24}=(0,1,0)$ and $\vec{d}_{34}=-\vec{d}_{12}$. The DM interaction and related spin structure in the orbital model (E1) are schematically shown in Fig. 17.

Spin canting and magnon gap: First we estimate anisotropy constants. We consider the orbital state with $n_{c}=0.6$ which has a chance to explain the $J^{(c)}=J^{(a b)}$ property, giving isotropic spin coupling $J$ about $-0.1 J_{S E}$ at $\eta=0.24$ (see Fig. 17). Given these parameters, one obtains from Eqs. (E7) and (E11),

$$
\begin{aligned}
\widetilde{A} & \simeq 11\left(\lambda / \Delta_{\alpha \beta}\right)^{2}|J|, \\
\widetilde{D}^{(c)} & \simeq 10\left(\lambda / \Delta_{\alpha \beta}\right)|J|, \\
\widetilde{D}^{(a b)} & =(3 / 2) \widetilde{D}^{(c)} .
\end{aligned}
$$

By minimizing classical energy of DM and isotropic Heisenberg interactions, we find that spins cant away from the $c$ axis by an angle $\theta \sim \frac{\widetilde{D}^{(a b)}}{3 \sqrt{2} J} \sim 3.5\left(\lambda / \Delta_{\alpha \beta}\right)$. Also, within a linear spin-wave theory we estimate a magnon gap generated by symmetric anisotropy and DM interactions as $\sqrt{3} \widetilde{A} S \sim 9.5\left(\lambda / \Delta_{\alpha \beta}\right)^{2}|J|$ and $4 S|J| \theta^{2} \sim$ $25\left(\lambda / \Delta_{\alpha \beta}\right)^{2}|J|$, correspondingly. With $\lambda \simeq 19 \mathrm{meV}$ and $|J| \simeq 2.75 \mathrm{meV}$, we obtain that the ratio $\left(\lambda / \Delta_{\alpha \beta}\right)$ must be less than 0.05-0.06 in order to be consistent with the observed canting angle $(\sim 0.17 \mathrm{rad})$ and an upper limit for the gap $(\sim 0.3 \mathrm{meV})$. Thus, the splitting of the lowest orbital doublet $\Delta_{\alpha \beta}$ should be at least about $300 \mathrm{meV}$. However, this is hard to reconcile with almost equal four short Ti-O bonds in titanates suggesting an almost degenerate doublet picture.

Based on the above analysis, we think that the orbital state (E1) predicted by band-structure calculations is not supported by recent neutron-scattering experiments in $\mathrm{YTiO}_{3} .{ }^{10}$ This is perhaps not really surprising, as a Mott insulator with orbital degeneracy represents a strongly correlated system, which is difficult to address in a framework of weakly interacting electrons.

* Present address: Department of Physics, Columbia University, 538 West 120th Street, New York, New York 10027.

${ }^{1}$ M. Imada, A. Fujimori, and Y. Tokura, Rev. Mod. Phys. 70, 1039 (1998).

${ }^{2}$ Y. Tokura and N. Nagaosa, Science 288,462 (2000).

${ }^{3}$ B. Keimer, D. Casa, A. Ivanov, J. W. Lynn, M. v. Zimmermann, J. P. Hill, D. Gibbs, Y. Taguchi, and Y. Tokura, Phys. Rev. Lett. 85, 3946 (2000).

${ }^{4}$ G. Khaliullin and S. Maekawa, Phys. Rev. Lett. 85, 3950 (2000).

${ }^{5}$ G. Khaliullin, P. Horsch, and A. M. Oleś, Phys. Rev. Lett. 86, 3879 (2001).

${ }^{6}$ K. I. Kugel and D. I. Khomskii, Sov. Phys. Usp. 25, 231 (1982).

${ }^{7}$ K. I. Kugel and D. I. Khomskii, Sov. Phys. Solid State 17, 285 (1975).

8 T. Katsufuji, Y. Taguchi, and Y. Tokura, Phys. Rev. B 56, 10145 (1997).

${ }^{9}$ J. P. Goral, J. E. Greedan, and D. A. MacLean, J. Solid State Chem. 43, 244 (1982).

${ }^{10}$ C. Ulrich, G. Khaliullin, S. Okamoto, M. Reehuis, A. Ivanov, H. He, Y. Taguchi, Y. Tokura, and B. Keimer, Phys. Rev. Lett. 89, 167202 (2002). 
${ }^{11}$ J. B. Goodenough, Phys. Rev. 100, 564 (1955).

12 J. Kanamori, J. Phys. Chem. Solids 10, 87 (1959).

${ }^{13}$ G. Khaliullin, Phys. Rev. B 64, 212405 (2001).

${ }^{14}$ G. Khaliullin and S. Okamoto, Phys. Rev. Lett. 89, 167201 (2002).

15 T. Mizokawa and A. Fujimori, Phys. Rev. B 54, 5368 (1996).

${ }^{16}$ In addition to the result of Ref. 13 , the operator $B_{i j}^{(\gamma)}$ in Eq. (5) contains more (last two) terms, which result from spin-singlet electron pair dynamics in the virtual $d^{2}$ excited state. It is noticed that such pair-hopping terms vanish in a spin polarized ferromagnetic state.

${ }^{17}$ S. Ishihara, T. Hatakeyama, and S. Maekawa, Phys. Rev. B 65, 064442 (2002).

18 Shun-Qing Shen, X. C. Xie, and F. C. Zhang, Phys. Rev. Lett. 88, 027201 (2002).

${ }^{19}$ For a discussion of the order-from-disorder phenomena in frustrated systems, see A. M. Tsvelik, Quantum Field Theory in Condensed Matter Physics (Cambridge University Press, Cambridge, 1995), Chap. 17, and references therein.

${ }^{20}$ V. N. Popov, Functional Integrals in Quantum Field Theory and Statistical Physics (Reidel, Dordrecht, 1983).

${ }^{21}$ M. Mochizuki and M. Imada, J. Phys. Soc. Jpn. 70, 1777 (2001).

${ }^{22}$ T. Moriya, Phys. Rev. 120, 91 (1960).

${ }^{23}$ H. Sawada, N. Hamada, and K. Terakura, Physica B 237238, 46 (1997); H. Sawada and K. Terakura, Phys. Rev. B 58, 6831 (1998).

${ }^{24}$ J. Akimitsu, H. Ichikawa, N. Eguchi, T. Miyano, M. Nishi, and K. Kakurai, J. Phys. Soc. Jpn. 70, 3475 (2001).

${ }^{25}$ M. Itoh, M. Tsuchiya, H. Tanaka, and K. Motoya, J. Phys. Soc. Jpn. 68, 2783 (1999).

${ }^{26}$ H. Nakao, Y. Wakabayashi, T. Kiyama, Y. Murakami, M. v. Zimmermann, J. P. Hill, D. Gibbs, S. Ishihara, Y. Taguchi, and Y. Tokura, Phys. Rev. B 66, 184419 (2002).

${ }^{27} \mathrm{~S}$. Okamoto and G. Khaliullin (unpublished).

${ }^{28}$ T. Yildirim, A. B. Harris, Amnon Aharony, and O. EntinWohlman, Phys. Rev. B 52, 10239 (1995).

${ }^{29}$ R. P. Feynman, Statistical Mechanics (Benjamin, New York, 1972), Chap. 11.

${ }^{30}$ A. Abragam and B. Bleaney, Electron Paramagnetic Resonance of Transition Ions (Oxford University Press, New York, 1970).

${ }^{31}$ Y. Murakami, H. Kawada, H. Kawata, M. Tanaka, T. Arima, Y. Moritomo, and Y. Tokura, Phys. Rev. Lett. 80, 1932 (1998).

32 S. Ishihara and S. Maekawa, Phys. Rev. Lett. 80, 3799 (1998).

${ }^{33}$ M. Takahashi and J. Igarashi, Phys. Rev. B 64, 075110 (2001).

${ }^{34}$ Y. Furukawa, I. Okamura, K. Kumagai, Y. Taguchi and Y. Tokura, Physica B 237-238, 39 (1997).

${ }^{35}$ S. Okubo, S. Kimura, H. Ohta, and M. Itoh, J. Magn. Magn. Mater. 177-181, 1373 (1998). 